\title{
LAS MONOCOTILEDÓNEAS NATIVAS DEL CORREDOR BIOLÓGICO CHICHINAUTZIN
}

\author{
Valeria Angélica Pulido-Esparza, Adolfo Espejo-Serna y Ana Rosa López- \\ FERRARI \\ Universidad Autónoma Metropolitana-Iztapalapa, Departamento de Biología, \\ Herbario Metropolitano, Apdo. postal. 55-535, 09340 México, D.F., México. \\ feliscatus@yahoo.com, aes@xanum.uam.mx
}

\section{RESUMEN}

El Corredor Biológico Chichinautzin es una Área Natural Protegida que cuenta con una extensión de 65,901 ha. En la zona se presenta una amplia variación ambiental y se encuentran representados siete tipos de vegetación en un intervalo altitudinal que va de 1200 a $3400 \mathrm{~m}$. Mediante la revisión de ejemplares de herbario, de bibliografía especializada y de recolección de material botánico, se obtuvo el listado de las monocotiledóneas nativas que crecen dentro de sus límites, que incluye 359 especies, 132 géneros y 25 familias. Sobresalen las Orchidaceae y Poaceae por su alto número de taxa; entre los grupos con plantas de distribución restringida destacan las familias Orchidaceae y Bromeliaceae. Dentro del área se encuentran seis elementos endémicos exclusivos.

Palabras clave: catálogo de monocotiledóneas, endemismo, México, Morelos, riqueza florística.

\begin{abstract}
The Corredor Biológico Chichinautzin is a natural protected area with an extension of 65,901 ha. Within its boundaries seven vegetation types are represented in an altitudinal range from 1200 to $3400 \mathrm{~m}$. Revision of herbarium collections and bibliography, and field specimens collections, let us to obtain the inventory of native monocots species present in the area. The inventory comprises 359 species, 132 genera and 25 families. Orchidaceae and Bromeliaceae stand out by their endemism level. In the area grow six exclusive species.
\end{abstract}

Key words: endemism, floristic richness, Mexico, Morelos, Monocots catalogue. 


\section{INTRODUCCIÓN}

El Corredor Biológico Chichinautzin (CBC), fue declarado área natural protegida (ANP) en 1988 (Anónimo) y a pesar de esto es una zona con un fuerte impacto antrópico, debido a su cercanía a las ciudades de México y Cuernavaca y a la creciente expansión de poblaciones y de áreas manejadas por el hombre.

Aunque se trata de una zona fuertemente perturbada, cuenta todavía con partes bien conservadas pero biológicamente poco conocidas, ya que no existen inventarios completos y actualizados de sus recursos naturales. Con el fin de subsanar al menos parcialmente tal situación, se planteó como objetivo primordial de este trabajo elaborar el catálogo de las especies de monocotiledóneas que crecen en la zona, así como determinar sus niveles de endemismo.

Las Liliopsideae constituyen un grupo bien representado a nivel nacional, con un total cercano a las 4424 especies nativas (Espejo et al., 2004), lo que equivale a $18.2 \%$ del total de angiospermas que crecen en el país. Entre las contribuciones al conocimiento de la flora y/o la vegetación de la zona de estudio podemos citar los trabajos de Hernández (1945); Ramírez-Cantú (1949); Reko (1954); Dressler (1960); Espinosa (1962); Rowell (1964); Corona (1967); Vázquez-Sánchez (1974); BonillaBarbosa y Novelo (1995); Bonilla-Barbosa y Viana (1997); Espejo et al. (1998); Cerros-Tlatilpa y Espejo (1998); Bonilla-Barbosa et al. (2000); Espejo et al. (2002) y Bonilla-Barbosa y Villaseñor (2003). Sin embargo, éste es el primero en el que se presenta el inventario integral de un grupo botánico para el área del CBC.

\section{ÁREA DE ESTUDIO}

El Corredor Biológico Chichinautzin ocupa la porción noroccidental del estado de Morelos, comprendiendo porciones de los municipios de Huitzilac, Cuernavaca, Tepoztlán, Tlayacapan, Jiutepec, Yautepec, Tlalnepantla y Totolapan, además de una parte del de Ocuilan de Arteaga en el Estado de México y sectores de las delegaciones Tlalpan y Milpa Alta en el Distrito Federal (Anónimo, 1988; 2002) (Fig. 1).

La región se encuentra catalogada como Área Natural Protegida y está constituida por dos fracciones correspondientes al Área de Protección de Flora y Fauna Silvestres Corredor Biológico Chichinautzin, y por dos parques nacionales (Lagunas de Zempoala y El Tepozteco).

El CBC cuenta con tres zonas núcleo: a) Chalchihuites, ubicada al norte del corredor con una superficie de 783 ha, y cuyo nombre alude al volcán situado en el 
extremo noroeste del área; b) Chichinautzin-Quiahuistepec, con 2873 ha, situada en la porción central de la zona de estudio, en donde se encuentran el volcán Chichinautzin y la loma Quiahuistepec; y c) Las Mariposas, que con una extensión de 1740 ha, ocupa el cerro del mismo nombre en la porción oriental de la Sierra de Tepoztlán (Aguilar-Benítez, 1995; Anónimo, 1988).

Dentro de los límites del CBC se desarrollan siete tipos de vegetación (sensu Rzedowski, 1978): bosque de coníferas (BC), bosque de encino (BQ), bosque mesófilo de montaña (BMM), bosque tropical caducifolio (BTC), matorral xerófilo (MX),
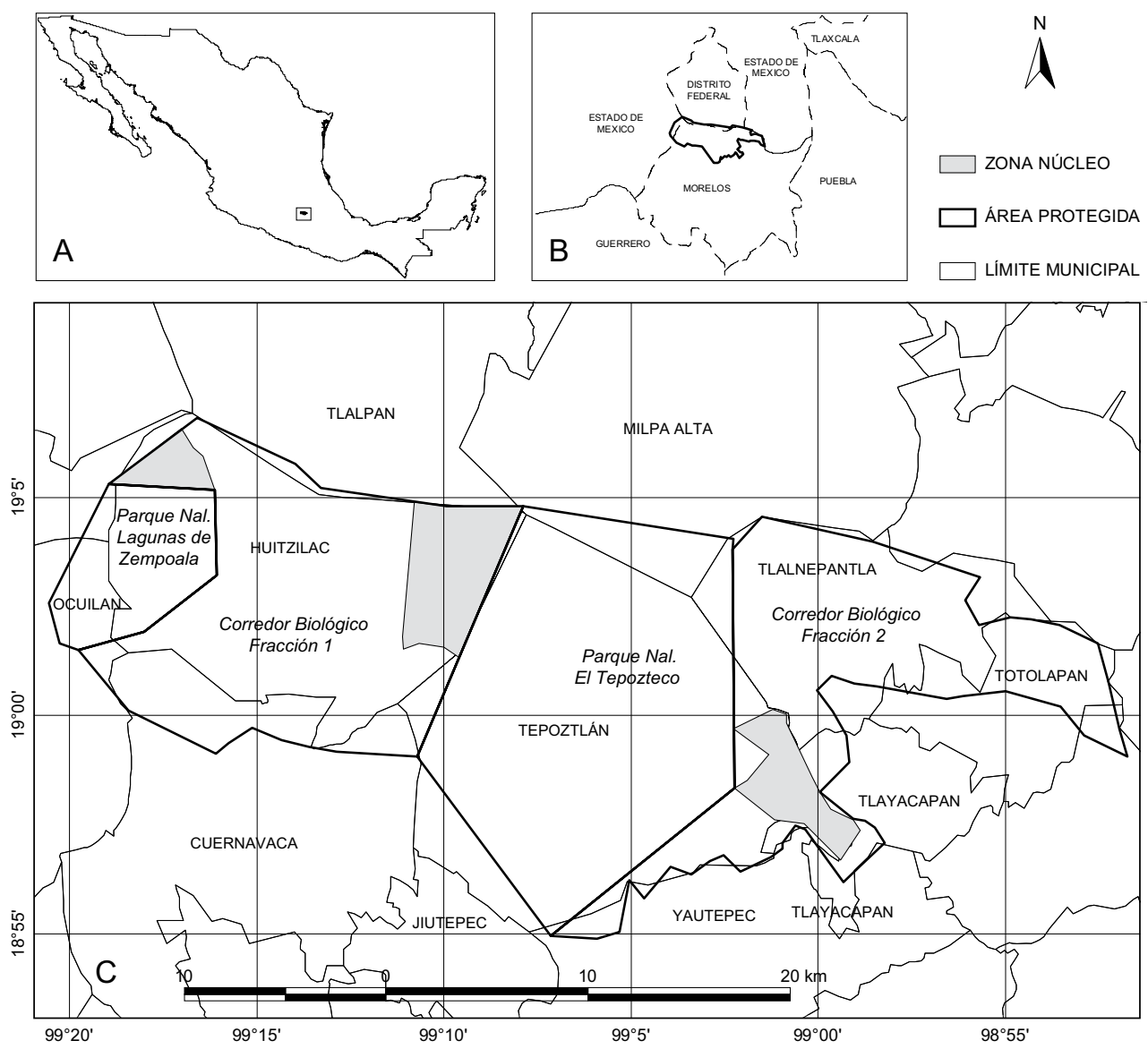

Fig. 1. Corredor Biológico Chichinautzin. A: ubicación de la zona en el país, B: ubicación de la zona a nivel estatal, C: polígono representando la conformación del área de estudio. 
pastizal (PZ) y vegetación acuática y subacuática (VAS), en un intervalo altitudinal que va de 1200 a $3400 \mathrm{~m}$.

\section{MÉTODOS}

Para la elaboración del catálogo se estudiaron 1354 ejemplares herborizados, depositados en las colecciones institucionales mexicanas AMO (Herbario de la Asociación Mexicana de Orquideología), ENCB (Herbario de la Escuela Nacional de Ciencias Biológicas, Instituto Politécnico Nacional), HUMO (Herbario de la Universidad Autónoma del Estado de Morelos), MEXU (Herbario Nacional del Instituto de Biología, Universidad Nacional Autónoma de México) y UAMIZ (Herbario Metropolitano Ramón Riba y Nava Esparza, Universidad Autónoma Metropolitana Iztapalapa) y de algunas colecciones históricas de los herbarios AMES (Herbario Oakes Ames, Harvard University) y F (Herbario del Field Museum of Natural History). También se llevó a cabo una revisión bibliográfica detallada sobre el tema y en diferentes momentos, durante los años 1996 a 2004, se visitaron diversas localidades del CBC con el fin de recolectar ejemplares botánicos de monocotiledóneas nativas, obteniéndose material de 90 especies, las cuales están incluidas en los análisis aquí presentados. La colecta y preservación de los especímenes se realizó de acuerdo con los métodos convencionales dados a conocer en la literatura (Lot y Chiang, 1986; Forman y Bridson, 1989). El primer juego de los ejemplares recolectados fue depositado en el herbario UAMIZ.

La información recabada se ordenó en una base de datos creada en una hoja de cálculo de Microsoft Excel, la cual incluyó los siguientes campos: familia, género, especie, estado, municipio, localidad, altitud, tipo de vegetación, datos ambientales, georeferencia, colector y número de colecta. En los casos en los cuales las etiquetas de los ejemplares revisados no contaban con las coordenadas geográficas de los sitios de recolección, se utilizaron cartas topográficas escala 1:50,000 para obtenerlos.

La riqueza y el endemismo se analizaron numéricamente y en relación con el ambiente, considerando la elevación sobre el nivel del mar y el tipo de vegetación. La información altitudinal obtenida de las etiquetas de los ejemplares se graficó por intervalos de 100 metros a partir de la mínima registrada, para conocer el número de especies y obtener su patrón de distribución. Lo mismo se hizo para los tipos de vegetación que se desarrollan en la zona, lo que nos permitió conocer la riqueza de especies en cada uno de ellos. 


\section{RESULTADOS Y DISCUSIÓN}

Riqueza

Dentro del área del Corredor Biológico Chichinautzin crecen 359 especies de monocotiledóneas nativas, agrupadas en 132 géneros y 25 familias (sensu Dahlgren et al., 1985). El catálogo completo se presenta en el apéndice e incluye la cita de al menos un ejemplar de respaldo para cada uno de los taxa enlistados.

Sobresalen las Orchidaceae con 130 especies y 44 géneros y las Poaceae con 75 y 38 respectivamente, lo que en conjunto suma $57.1 \%$ del total de las monocotiledóneas existentes en el área. Del resto de las familias, cinco figuran con sólo un representante, ocho cuentan con un género pero con varios taxa y las 10 restantes conforman un grupo heterogéneo en cuanto a su representación numérica en la zona (Cuadro 1).

En la figura 2, relativa a la distribución de las especies a lo largo de los intervalos altitudinales establecidos, se observa una mayor concentración de éstas entre 1700 y 1800 m, seguida por aquellas ubicadas entre 2100 y 2200 m, para incrementarse nuevamente entre 2800 y $3000 \mathrm{~m}$.

Al analizar dicha repartición destaca el comportamiento peculiar del tercer pico, correspondiente a $2800 \mathrm{~m}$, donde se refleja un aumento en la riqueza específica asociada directamente con los tipos de vegetación predominantes en esas altitudes, es decir, los bosques de coníferas, los pastizales y las comunidades acuáticas.

Uniendo los dos primeros picos altitudinales se podría definir un intervalo de 500 metros en el cual se ubica la mayor cantidad de especies presentes en la zona, asociada con los tipos de vegetación que ahí se desarrollan, que corresponden a seis de los siete registrados para el área de estudio. Considerando estos resultados es posible relacionar la gran riqueza de hábitats encontrada entre 1700 y $2200 \mathrm{~m}$ con la de las monocotiledóneas que crecen en el área. Podemos ubicar más precisamente estos sitios en el sector central del CBC, en el municipio de Tepoztlán y en algunas porciones aledañas a los de Huitzilac, Tlalnepantla y Tlayacapan.

Con respecto a la distribución de las especies en los distintos tipos de vegetación, se observó que el mayor número prospera en los bosques de coníferas, seguidos por los tropicales caducifolios y los de Quercus (Fig. 3).

El CBC se ubica en la parte meridional del Eje Volcánico Transmexicano y consecuentemente dentro de sus límites se desarrollan extensiones considerables de bosques de coníferas, especialmente en la porción norte. Este sistema montañoso constituye la cordillera más alta del país y los elementos florísticos que la componen tienen orígenes muy diferentes, en función de la transición entre dos zonas 
biogeográficas: la Neártica y la Neotropical (Almeida et al., 1990; Mardocheo et al., 2001).

Se ha planteado en reiteradas ocasiones la influencia de las condiciones físicas y ecológicas del Eje Volcánico Transmexicano en los patrones de distribución de los

Cuadro 1. Número total de géneros y especies de monocotiledóneas presentes en el Corredor Biológico Chichinautzin.

\begin{tabular}{lcc}
\hline Familia & Número de géneros & Número de especies \\
\hline Agavaceae & 2 & 7 \\
Alliaceae & 4 & 4 \\
Alstromeriaceae & 1 & 1 \\
Amaryllidaceae & 3 & 6 \\
Anthericaceae & 1 & 9 \\
Araceae & 2 & 2 \\
Bromeliaceae & 4 & 19 \\
Calochortaceae & 1 & 4 \\
Commelinaceae & 8 & 21 \\
Cyperaceae & 7 & 28 \\
Dioscoreaceae & 1 & 12 \\
Eriocaulaceae & 1 & 3 \\
Hydrocharitaceae & 1 & 1 \\
Hypoxidaceae & 1 & 4 \\
Iridaceae & 3 & 12 \\
Juncaceae & 2 & 5 \\
Juncaginaceae & 1 & 1 \\
Lemnaceae & 1 & 2 \\
Melanthiaceae & 2 & 4 \\
Orchidaceae & 44 & 130 \\
Poaceae & 38 & 75 \\
Pontederiaceae & 1 & 1 \\
Potamogetonaceae & 1 & 359 \\
Smilacaceae & 1 & \\
Typhaceae & 1 & 1 \\
Total & & 1 \\
\hline
\end{tabular}




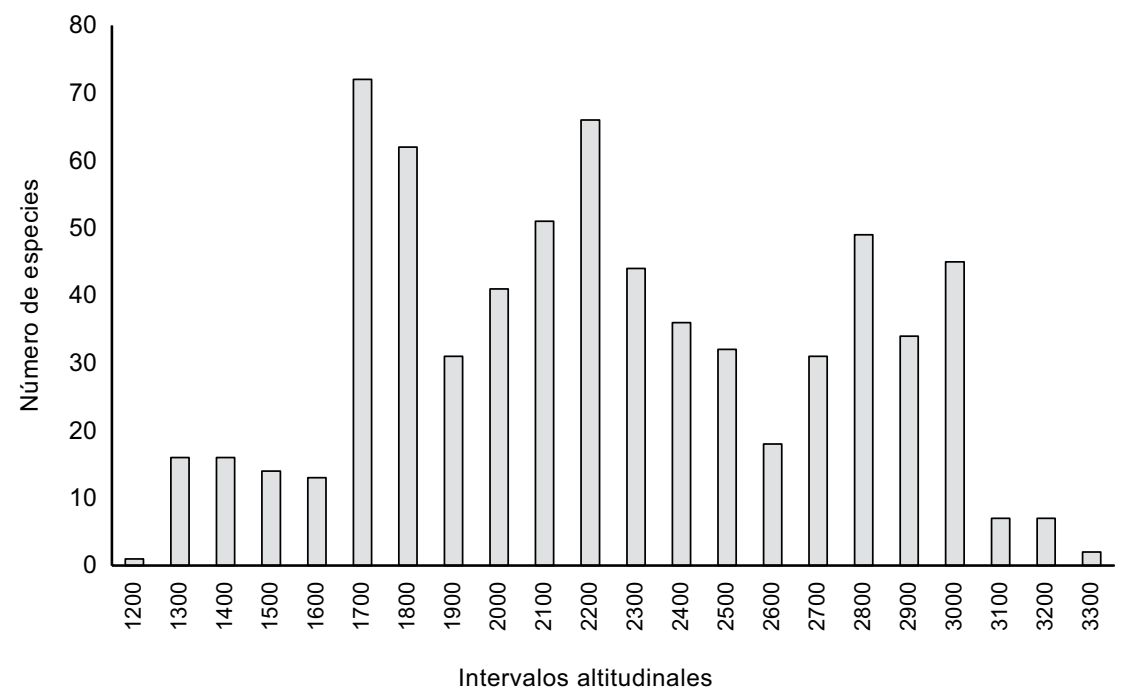

Fig. 2. Número de especies de monocotiledóneas en el CBC por intervalo altitudinal.

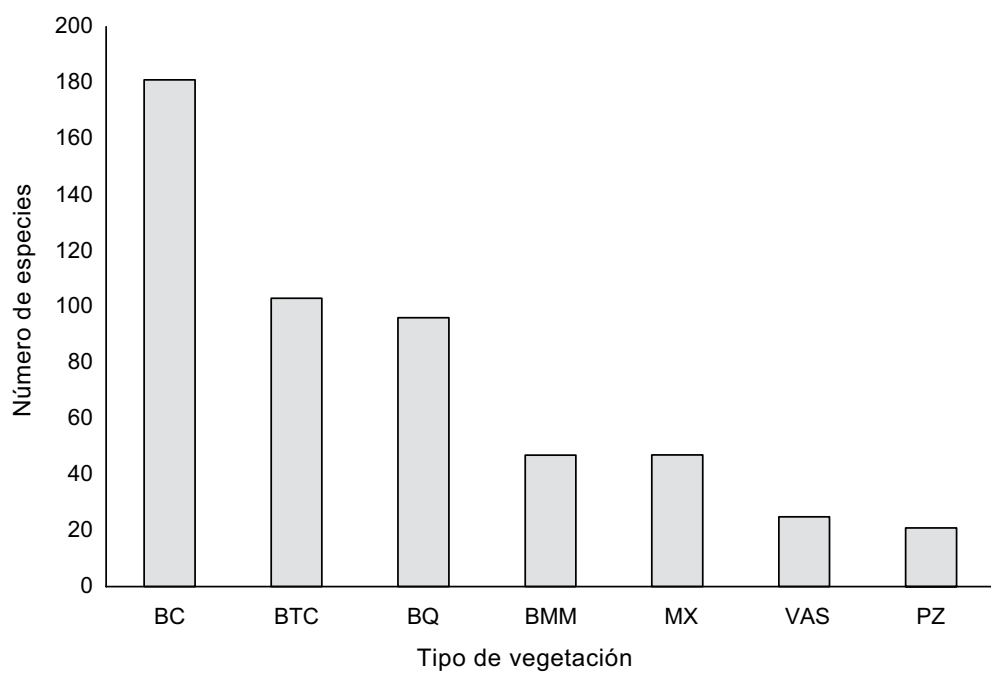

Fig. 3. Número de especies de monocotiledóneas por tipo de vegetación. BC: Bosque de coníferas; BTC: Bosque tropical caducifolio; BQ: Bosque de Quercus; BMM: Bosque mesófilo de montaña; MX: Matorral xerófilo; VAS: Vegetación acuática y subacuática; PZ: Pastizal. 
organismos, ya que favorece la existencia de gradientes climáticos, lo que aunado al origen geológico de este sistema orográfico y a su ubicación en la zona intertropical, ha permitido el desarrollo de numerosos ambientes que albergan especies de grupos muy diversos (Delgadillo, 2003).

\section{Endemismo}

El análisis del componente endémico de las monocotiledóneas del $\mathrm{CBC}$ se realizó a tres niveles; el primero agrupando a aquellas especies exclusivas de México cuya distribución abarca la zona de estudio. Dentro de este conjunto encontramos que el corredor alberga 100, pertenecientes a 48 géneros y 14 familias. Destaca nuevamente por su alto porcentaje la familia Orchidaceae con 41 spp., seguida por las Bromeliaceae con 12. Las doce familias restantes tienen un número bajo de componentes restringidos al territorio nacional (Cuadro 2).

Cuadro 2. Elementos endémicos de las monocotiledóneas en el Corredor Biológico Chichinautzin.

\begin{tabular}{|c|c|c|c|c|}
\hline Familia & $\begin{array}{l}\text { Número de } \\
\text { especies totales } \\
\text { en el CBC }\end{array}$ & $\begin{array}{l}\text { Número de } \\
\text { especies } \\
\text { endémicas a } \\
\text { nivel nacional }\end{array}$ & $\begin{array}{l}\text { Número de } \\
\text { especies } \\
\text { endémicas a } \\
\text { nivel estatal }\end{array}$ & $\begin{array}{l}\text { Número de } \\
\text { especies } \\
\text { endémicas a nivel } \\
\text { local (CBC) }\end{array}$ \\
\hline Orchidaceae & 130 & $41 \quad(31.5 \%)$ & $4 \quad(3.07 \%)$ & $3(2.3 \%)$ \\
\hline Cyperaceae & 28 & $1 \quad(3.5 \%)$ & $1 \quad(3.5 \%)$ & $1(3.5 \%)$ \\
\hline Commelinaceae & 21 & $7 \quad(33.3 \%)$ & - & - \\
\hline Bromeliaceae & 19 & $12(63.15 \%)$ & $1 \quad(5.26 \%)$ & - \\
\hline Dioscoreaceae & 12 & $8 \quad(66.6 \%)$ & $2(16.6 \%)$ & - \\
\hline Iridaceae & 12 & $7 \quad(58.33 \%)$ & $2(16.6 \%)$ & $1(8.3 \%)$ \\
\hline Anthericaceae & 9 & $6 \quad(66.6 \%)$ & - & - \\
\hline Agavaceae & 7 & $5 \quad(71.42 \%)$ & - & - \\
\hline Calochortaceae & 4 & $4 \quad(100 \%)$ & - & - \\
\hline Melanthiaceae & 4 & $3(75 \%)$ & $2(50 \%)$ & $1(25 \%)$ \\
\hline Smilacaceae & 4 & $3(75 \%)$ & - & - \\
\hline Eriocaulaceae & 3 & $1 \quad(33.3 \%)$ & - & - \\
\hline Hypoxidaceae & 4 & $1 \quad(25 \%)$ & - & - \\
\hline Araceae & 2 & $1 \quad(50 \%)$ & - & - \\
\hline Total endemitas & & $100 \quad(27.85 \%)$ & $12 \quad(3.3 \%)$ & $6(1.67 \%)$ \\
\hline
\end{tabular}


El segundo nivel agrupa a las especies endémicas de alguno de los estados que incluye la zona de estudio. Para el caso de Morelos, se tienen doce, comprendidas en 9 géneros y 6 familias.

La tercera categoría corresponde a los taxa que son exclusivos del CBC, y en este rubro se cuenta con seis, agrupados en 6 géneros y 4 familias. Dichos elementos son: Carex interjecta Reznicek, Tigridia tepoxtlana Ravenna, Schoenocaulon tenue Brinker, Malaxis palustris Espejo \& López-Ferrari, Pleurothallis nigriflora L.O. Williams y Ponera dressleriana Soto Arenas.

Sobresale en los tres niveles la familia Orchidaceae, con 41 especies endémicas nacionales y cuatro estatales de las cuales tres son también endemitas locales. En el cuadro 2 se muestran los porcentajes de representación de los elementos con distribución restringida a nivel nacional, estatal y local para cada familia.

El total de los taxa de Calochortaceae encontrados en el CBC, son exclusivos de México, así como 75\% de los de las Melanthiaceae y 71.4\% de los de las Agavaceae; aun cuando el número de endemitas de estas familias es bajo (4, 3 y 5 especies respectivamente), corresponden a un alto porcentaje de representación con respecto al total registrado para el corredor (4, 4 y 7 respectivamente). En cuanto a los niveles estatal y local, destaca nuevamente la familia Melanthiaceae con 50 y $25 \%$ respectivamente.

En lo referente a la distribución altitudinal de las especies endémicas del país presentes en el CBC, se encuentra una tendencia similar a la observada para el caso de la riqueza general, ya que el mayor número de taxa se ubica entre 1700 y $1800 \mathrm{~m}$, y entre 2200 y $2500 \mathrm{~m}$. (Fig. 4).

Los tipos de vegetación en los que está representada la mayor cantidad de endemismos vuelven a ser los bosques de coníferas, seguidos por los de Quercus y los bosques tropicales caducifolios, de manera muy similar a lo que se encontró en el análisis de la riqueza (Fig. 5).

\section{CONCLUSIONES}

La riqueza de monocotiledóneas en el Corredor Biológico Chichinautzin asciende a $8.18 \%$ del total registrado a nivel nacional por Espejo et al. (2004), quienes reconocen 4,424 especies de Liliopsida para México. Los resultados obtenidos indican un alto porcentaje de representación, considerando la gran cantidad de taxa encontrados en una zona relativamente pequeña $(65,901 \mathrm{ha})$. En consecuencia el área reviste un interés particular desde el punto de vista biológico y por lo tanto es im- 


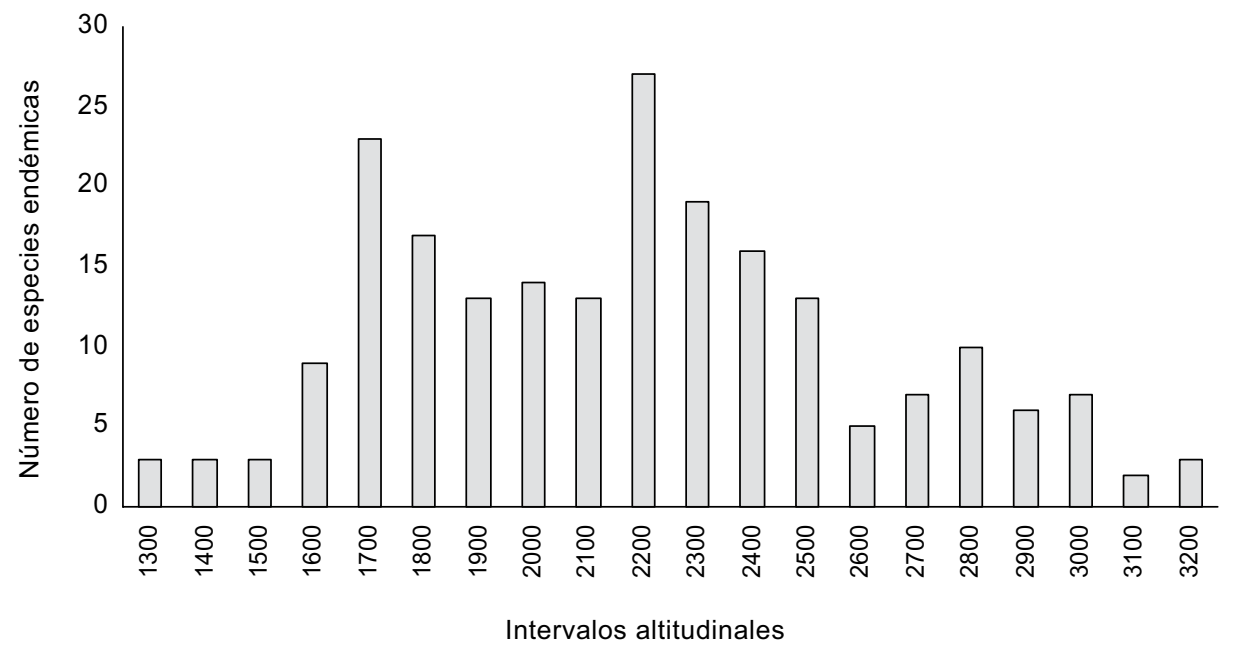

Fig. 4. Número de especies endémicas de monocotiledóneas del CBC por intervalo altitudinal (1300 a $3200 \mathrm{~m})$.

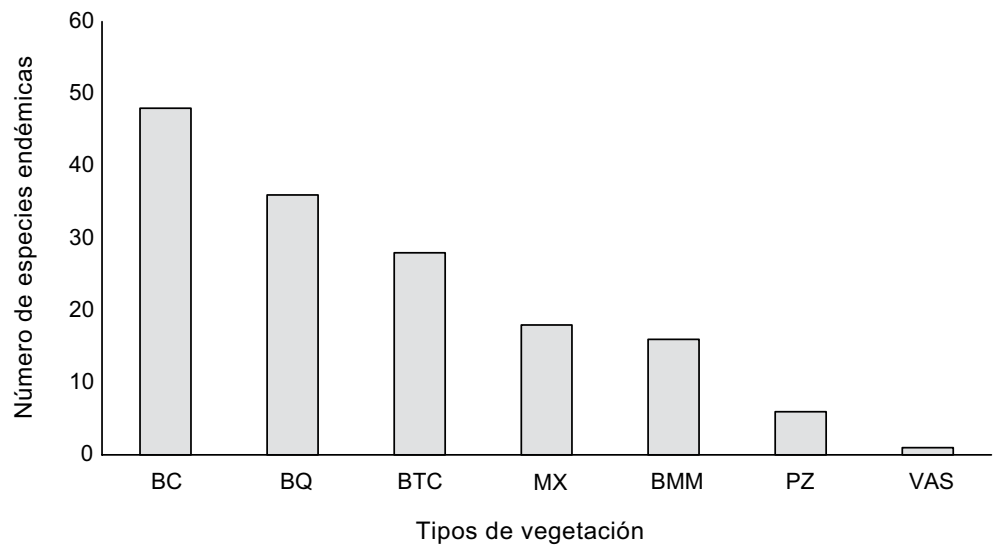

Fig. 5. Número de especies endémicas de monocotiledóneas del CBC por tipo de vegetación. BC: Bosque de coníferas; BQ: Bosque de Quercus; BTC: Bosque tropical caducifolio; MX: Matorral xerófilo; BMM: Bosque mesófilo de montaña; PZ: Pastizal; VAS: Vegetación acuática y subacuática. 
portante conservarla y protegerla. En el Parque Nacional Lagunas de Zempoala, las comunidades acuáticas se han visto severamente afectadas por la extracción de agua para abastecer diversas poblaciones aledañas, como Huitzilac y Tres Marías, en el estado de Morelos y Santa María Ocuilan, en el Estado de México (Bonilla-Barbosa y Novelo, 1995). Del mismo modo, la Sierra de Tepoztlán se ha ido poblando paulatinamente, con el consecuente deterioro en los hábitats naturales que conlleva la presencia de los asentamientos humanos.

Finalmente cabe señalar que, de acuerdo con los datos disponibles, ninguno de los endemitas restringidos al CBC se encuentra en las zonas núcleo, por lo que sus poblaciones podrían ser más vulnerables a los efectos antrópicos cada vez más severos.

\section{AGRADECIMIENTOS}

Agradecemos a Alejandro Flamenco y a Alejandro Zavala la revisión crítica del manuscrito, así como sus atinados comentarios y sugerencias. Asimismo expresamos nuestra gratitud a los encargados de los herbarios consultados que nos brindaron todas las facilidades para la revisión del material. También agradecemos el apoyo en el trabajo de campo de los biólogos Marco Antonio Pulido-Giles, Jorge Santana-Carrillo y Ezequiel Mora-Guzmán. Los mapas se realizaron con la ayuda de Alejandro Flamenco. Los resultados de este trabajo forman parte de la tesis de Maestría en Biología de la primera autora que contó con el apoyo del Consejo Nacional de Ciencia y Tecnología a través de la beca 164710.

\section{LITERATURA CITADA}

Aguilar-Benítez, S. 1995. Ecología del estado de Morelos. Un enfoque geográfico. Ed. Praxis. Cuernavaca. $469 \mathrm{pp}$.

Almeida, L., I. Luna y A. Herrera. 1990. Método de estudio integral de las comunidades vegetales de la región central del Eje Neovolcánico. In: Camarillo, J. L. y F. Rivera (comps.). Áreas naturales protegidas en México y especies en extinción. Proyecto Conservación y Mejoramiento del Medio Ambiente-Universidad Nacional Autónoma de México. México, D.F. 374 pp.

Anónimo. 1988. Decreto por el que se declara el Área de Protección de la Flora y Fauna Silvestres, ubicada en los municipios de Huitzilac, Cuernavaca, Tepoztlán, Jiutepec, Tlalnepantla, Yautepec, Tlayacapan y Totolapan, Morelos. Diario Oficial de la Federación. 30 de Noviembre de 1988. México. D.F. 
Anónimo. 2002. Anuario estadístico del estado de Morelos. Instituto Nacional de Estadística, Geografía e Informática. México. Aguascalientes. 492 pp.

Bonilla-Barbosa, J. R. y A. Novelo R. 1995. Manual de identificación de plantas acuáticas del Parque Nacional Lagunas de Zempoala, México. Cuadernos 26. Instituto de Biología, Universidad Nacional Autónoma de México. México, D.F. 168 pp.

Bonilla-Barbosa, J. R. y J. A. Viana. 1997. Listados florísticos de México XIV. Flora del Parque Nacional Lagunas de Zempoala, México. Instituto de Biología, Universidad Nacional Autónoma de México. México, D.F. 31 pp.

Bonilla-Barbosa, J. R., J. A. Viana y F. Salazar-Villegas. 2000. Listados florísticos de México XX. Flora acuática de Morelos. Instituto de Biología, Universidad Nacional Autónoma de México. México, D.F. 29 pp.

Bonilla-Barbosa, J. R. y J. L. Villaseñor R. 2003. Catálogo de la flora del estado de Morelos. Centro de Investigaciones Biológicas, Universidad Autónoma del Estado de Morelos. Cuernavaca. 129 pp.

Cerros-Tlatilpa, R. y A. Espejo. 1998. Contribución al estudio florístico de los cerros El Sombrerito y Las Mariposas (Zoapapalotl), municipio de Tlayacapan, Morelos, México. Polibotánica 8: 29-46.

Corona, V. 1967. Introducción al estudio de la flora de los alrededores de Cuernavaca, Morelos. Tesis de licenciatura. Facultad de Ciencias, Universidad Nacional Autónoma de México. México, D.F. 67 pp.

Dahlgren, R. M. T., H. T. Clifford y P. F. Yeo. 1985. The families of the Monocotyledons. Springer Verlag. Berlín. 520 pp.

Delgadillo, C. 2003. Patrones biogeográficos de los musgos de México. In: Morrone, J. y J. Llorente (eds.). Una perspectiva latinoamericana de la biogeografía. Facultad de Ciencias, Universidad Nacional Autónoma de México. México, D.F. 307 pp.

Dressler, R. L. 1960. Tepoztlán, México, interesting orchid locality. Orch. Dig. 24: 297299.

Espejo, A., A. R. López-Ferrari, J. García-Cruz, R. Jiménez-Machorro y L. Sánchez-Saldaña. 1998. Les Orchidées du Couloir Biologique Chichinautzin. Orchidées, Culture et Protection 34: 9-11, 36.

Espejo, A., J. García-Cruz, A. R. López-Ferrari, R. Jiménez-Machorro y L. SánchezSaldaña. 2002. Orquídeas del Estado de Morelos. Orquídea (Méx.) 16: 1-332.

Espejo, A., A. R. López-Ferrari e I. Salgado-Ugarte. 2004. A current estimate of angiosperm diversity in Mexico. Taxon 53: 127-130.

Espinosa, J. 1962. Vegetación de una corriente de lava de formación reciente localizada en el declive meridional de la Sierra del Chichinautzin. Bol. Soc. Bot. Méx. 27: 67-114.

Forman, L. y D. Bridson. 1989. The herbarium handbook. Royal Botanic Gardens. Kew. 214 pp.

Hernández, P. 1945. La flora maravillosa de Tepoztlán. Bol. Soc. Bot. Méx. 3: 13-15.

Lot, A. y F. Chiang (comps.) 1986. Manual de herbario. Administración y manejo de colecciones, técnicas de recolección y preparación de ejemplares de herbario. Consejo Nacional de la Flora de México, A.C. México, D.F. 142 pp.

Mardocheo, P., J. Romero y A. Velásquez. 2001. La Cuenca de México: una revisión de su importancia biológica. Biodiversitas 37: 12-15. 
Ramírez-Cantú, D. 1949. Notas generales sobre la vegetación de la Sierra de Tepoztlán, Morelos. Anales Inst. Biol. Univ. Nac. Autón. México 20: 189-228.

Reko, B. P. 1954. Nombres botánicos de algunas plantas de Tepoztlán, Morelos. Bol. Soc. Bot. Méx. 2: 17-18.

Rowell, M. 1964. Notes on the vegetation of the Mexican State of Morelos. Sida 1: 262268.

Rzedowski, J. 1978. Vegetación de México. Limusa. México, D.F. 432 pp.

Vázquez-Sánchez, J. 1974. Contribución al conocimiento de las plantas del Estado de Morelos (México). Ciencia 29: 1-138.

Recibido en enero de 2007. Aceptado en septiembre de 2008. 


\section{APÉNDICE}

Catálogo de las especies de monocotiledóneas nativas del Corredor Biológico Chichinautzin

Las familias y las especies se enlistan por orden alfabético y se señalan con un * aquellas que son endémicas de México, con ** las endémicas de alguno de los estados de México, Morelos o Distrito Federal y con *** las endémicas del área de estudio.

\section{AGAVACEAE}

Agave angustifolia Haw.: A. Pulido 90 (UAMIZ).

*Agave dasylirioides Jacobi \& Bouché: A. Garcia-Mendoza 6331 (MEXU), 6540 (MEXU); $H$. S. Gentry 1203 (MEXU), 19581 (MEXU), 22498 (MEXU); C. G. Pringle s.n. (MEXU). *Agave horrida Lem. ex Jacobi: E. Bobadilla 13 (UAMIZ); A. Espejo, 1738 (ENCB, UAMIZ); R. Medina 212 (UAMIZ).

*Agave inaequidens K. Koch: G. Barroso 23 (UAMIZ); A. Garcia- Mendoza 6139 (MEXU); A. Pulido 56 (UAMIZ).

*Manfreda pringlei Rose: A. Espejo 4591 (UAMIZ); A. García- Mendoza 6651 (MEXU); F. Morales s.n. (MEXU); R. Torres C. 14014 (MEXU).

*Manfreda pubescens (Regel \& Ortgies) Verh.-Will.: A. García- Mendoza 6116 (MEXU), 6904 (MEXU).

Manfreda scabra (Ortega) McVaugh: A. Espejo 2628 (MEXU, UAMIZ); M. Flores C. 13 (UAMIZ), 126 (UAMIZ).

\section{ALLIACEAE}

Allium glandulosum Link \& Otto: R. Cerros 5(UAMIZ); A. R. López-Ferrari 2508(UAMIZ), 2556 (UAMIZ); C. G. Pringle 9251 (MEXU); J. L. Villaseñor 948 (MEXU).

Bessera elegans Schult. \& Schult.f.: J. Ceja 80 (UAMIZ); R. Cerros 7 (UAMIZ); A. Espejo 3850 (UAMIZ), 5915 (UAMIZ), 6114 (UAMIZ); R. Jiménez M. 5915 (UAMIZ); A. R. López-Ferrari 1217 (UAMIZ); E. Lyonnet 500800050 (MEXU), 520900013 (MEXU), 521100016 (MEXU); D. Martínez A. s.n. (MEXU); J. L. Villaseñor 948 (MEXU).

Milla biflora Cav.: R. Cerros 205 (UAMIZ); A. Espejo 2541 (UAMIZ); A. R. López-Ferrari 1217 (UAMIZ).

Nothoscordum bivalve (L.) Britton: A. Espejo 3293 (UAMIZ), 3625 (UAMIZ); F. Miranda 231 (MEXU).

\section{ALSTROEMERIACEAE}

Bomarea hirtella (Kunth) Herb.: A. Bonfil C. 1007 (MEXU); G. Díaz P. s.n. (MEXU); A. Espejo 1192 (UAMIZ); A. Flores C. s.n. (MEXU); M. Flores C. 125 (UAMIZ); A. R. López-Ferrari 1224 (UAMIZ); E. Lyonnet 1000 (MEXU), 1489 (MEXU); D. Martínez A. 6905 (MEXU); R. Torres C. 14016 (MEXU); M. Sousa 4465 (MEXU); J. L. Villaseñor 950 (MEXU). 


\section{AMARYLLIDACEAE}

Hymenocallis glauca (Herb.) Baker ex Benth. \& Hook.f.: R. Cerros 200 (UAMIZ).

Hymenocallis graminifolia Greenm.: A. Espejo 6073 (UAMIZ).

Hymenocallis harrisiana Herb.: H. Cota 7808 (ENCB, MEXU); A. Espejo 2519 (UAMIZ), 3621 (UAMIZ), 4983 (UAMIZ, MEXU); E. R. García 26 (ENCB); A. R. López-Ferrari 1219 (UAMIZ); A. Pulido 9 (UAMIZ); 76 (UAMIZ); J. Rzedowski 19780 (ENCB).

Sprekelia formosissima (L.) Herb.: R. Cerros 291 (HUMO); A. Pulido 25 (UAMIZ).

Zephyranthes fosteri Traub: A. Pulido 3 (UAMIZ), 12 (UAMIZ), 35 (UAMIZ), 40 (UAMIZ), 62 (UAMIZ), 64 (UAMIZ).

Zephyranthes sessilis Herb.: A. R. López-Ferrari 2713 (UAMIZ).

\section{ANTHERICACEAE}

*Echeandia durangensis (Greenm.) Cruden: R. Galván 1365 (ENCB, UAMIZ); A. R. LópezFerrari 760 (ENCB, MEXU, UAMIZ).

*Echeandia echeandioides (Schltdl.) Cruden: J. Espinosa 149 (ENCB, MEXU).

Echeandia flavescens (Schult. \& Schult.f.) Cruden: A. Rodríguez C. 2784 (MEXU).

*Echeandia gracilis Cruden: A. Kenton 23 (MEXU); H. G. Quiram 33 (MEXU).

Echeandia longipedicellata Cruden: J. Bonilla 472 (HUMO); 736 (HUMO); M. Gutiérrez s.n. (MEXU); R. D. López 642 (MEXU).

*Echeandia mexicana Cruden: L. Abundiz 715 (MEXU); I. J. De la Cruz 1424 (UAMIZ); A. Espejo 3319 (UAMIZ).

*Echeandia paniculata Rose: G. Barroso 57 (UAMIZ); E. Cabrera 12027 (MEXU); A. Espejo 3340 (MEXU, UAMIZ); M. Flores C. 128 (MEXU); A. R. López-Ferrari 2033 (MEXU).

Echeandia reflexa (Cav.) Rose: C. G. Pringle s.n. (HUMO).

*Echeandia tenuis (Weath.) Cruden: A. Espejo 5942 (UAMIZ).

\section{ARACEAE}

Arisaema macrospathum Benth.: A. Espejo 2851 (UAMIZ), 3286 (UAMIZ), 3344 (UAMIZ), 4997 (UAMIZ), 5413 (UAMIZ), 6104 (UAMIZ); E. L. Estrada 1056(UAMIZ); A. R. LópezFerrari 2176 (UAMIZ); O. Moreno s.n. (HUMO); A. Pulido 86 (UAMIZ); G. Serrano J. 23 (UAMIZ).

*Syngonium neglectum Schott: R. Cerros 197 (UAMIZ).

\section{BROMELIACEAE}

*Hechtia matudae L.B. Sm.: A. Espejo 4998 (UAMIZ).

*Hechtia podantha Mez: A. Bonfil C. 1008 (MEXU); J. Espinosa s.n. (HUMO); M. Rojas s.n. (MEXU).

Pitcairnia heterophylla (Lindl.) Beer: M. Flores C. 27 (UAMIZ); J. Vázquez 5009 (MEXU).

*Pitcairnia karwinskyana Schult. \& Schult.f.: A. Bonfil C. 978 (MEXU); M. Ishiki 899 (MEXU); A. R. López-Ferrari 2449 (UAMIZ).

*Pitcairnia palmeri S. Watson: A. Espejo 6089 (UAMIZ); F. Miranda 177 (MEXU). 
*Pitcairnia pteropoda L.B. Sm.: J. Espinosa 88 (UAMIZ), 138 (UAMIZ); E. Guízar N. 2770 (UAMIZ); E. Matuda 26319 (UAMIZ); R. Medina 2 (UAMIZ); L. I. Nevling Jr. 341 (GH, MEXU); V. Sánchez 23 (UAMIZ); J. Vázquez 2449 (MEXU), 3264 (MEXU).

*Tillandsia andrieuxii (Mez) L.B. Sm.: E. L. Estrada 1307 (MEXU); A. R. López-Ferrari 2852 (UAMIZ).

*Tillandsia bourgaei Baker: J. Ceja 781 (UAMIZ); R. Cerros 236 (UAMIZ); P. Fryxell 2318 (ENCB); E. Matuda 38328 (MEXU), 38528 (MEXU); F. Miranda 178 (UAMIZ), 3859 (MEXU); J. Vázquez 3014 (MEXU).

Tillandsia caput-medusae E. Morren: M. Flores C. 92 (UAMIZ); E. R. García 188 (ENCB); A. R. López-Ferrari 2465 (UAMIZ), 2709 (UAMIZ); E. Matuda 26034 (MEXU); F. Miranda 1292 (MEXU); A. Pulido 89 (UAMIZ); B. P. Reko 4663 (MEXU).

**Tillandsia cryptantha Baker: A. Espejo 6032 (UAMIZ), 6093 (UAMIZ), 6095 (UAMIZ); E. L. Estrada 1776 (MEXU, UAMIZ); E. Matuda 38329 (MEXU).

Tillandsia fasciculata Sw.: A. Espejo 5921 (UAMIZ); D. Martinez A. 28852 (MEXU, UAMIZ); E. Matuda 26318 (MEXU); F. Miranda 1304 (MEXU).

Tillandsia ionantha Planch.: A. R. López-Ferrari 2462 (UAMIZ); J. Vázquez 1525 (MEXU).

*Tillandsia makoyana Baker: M. Flores C. 26(UAMIZ); A. R. López-Ferrari 2464 (UAMIZ), 2716 (UAMIZ); A. Pulido 26 (UAMIZ).

*Tillandsia prodigiosa (Lem.) Baker: A. Espejo 6029 (UAMIZ); A. R. López-Ferrari 2371 (UAMIZ).

Tillandsia recurvata (L.) L.: G. Barroso 29 (UAMIZ); G. Carrillo s.n. (ENCB); J. Espinosa 238 (ENCB, MEXU); A. R. López-Ferrari 2710 (UAMIZ); E. Matuda 21478 (MEXU); G. Serrano J. 46 (UAMIZ).

Tillandsia schiedeana Steud.: R. Cerros 191 (UAMIZ); A. R. López-Ferrari 2466 (UAMIZ); A. Pulido 93 (UAMIZ).

Tillandsia usneoides (L.) L.: R. Cerros 292 (HUMO); J. L. Gonzaga 10 (MEXU).

*Tillandsia violacea Baker: A. Espejo 5639 (UAMIZ); J. Espinosa 135 (MEXU); A. R. López-Ferrari 2853 (UAMIZ); E. Matuda 26361 (MEXU), 38330 (MEXU); A. Pulido 13 (UAMIZ), 27 (UAMIZ), 71 (UAMIZ), 72 (UAMIZ).

*Viridantha atroviridipetala (Matuda) Espejo: J. Ceja 782 (UAMIZ); A. Espejo 5005 (UAMIZ); J. Espinosa 237 (UAMIZ); M. Flores C. 380 (UAMIZ); F. Miranda 1307 (MEXU); A. Pulido 33 (UAMIZ), 91 (UAMIZ); J. Vázquez 3613 (MEXU).

\section{CALOCHORTACEAE}

*Calochortus barbatus (Kunth) J. H. Painter: D. Martínez A. s.n. (MEXU); M. Olvera 89 (MEXU).

*Calochortus cernuus J.H. Painter: A. Bonfil C. 1176 (MEXU); J. Ceja 69 (UAMIZ), 74 (UAMIZ); R. Cerros 22 (UAMIZ); A. Espejo 2622 (UAMIZ); A. R. López-Ferrari 1214 (UAMIZ), 2034 (MEXU, UAMIZ).

*Calochortus pringlei B.L. Rob.: G. Barroso 65 (UAMIZ); L. W. Boege 1988 (MEXU); E. Lyonnet 2561 (MEXU); M. Ortiz O. 146 (MEXU). 
*Calochortus purpureus (Kunth) Baker: J. Flores 47 (ENCB); D. Martínez A. s.n. (MEXU); A. Pérez s.n. (MEXU).

\section{COMMELINACEAE}

Commelina coelestis Willd.: A. Bonfil C. s.n. (MEXU); R. Cerros 78 (UAMIZ); I. Díaz V. 1073 (MEXU, HUMO); J. M. Díaz 151 (ENCB); C. Dueñas 99 (ENCB); A. Espejo 1196 (ENCB, UAMIZ); D. B. Gold 193 (MEXU); F. W. Gould 193 (ENCB); H. E. Iñiguez s.n. (ENCB); A. Kenton 33 (MEXU); G. Manzanero 1178 (MEXU); R. Monroy M. (MEXU); A. Vargas N. s.n. (ENCB).

Commelina dianthifolia Delile: J. Espinosa 4 (ENCB); D. B. Gold 186 (ENCB, MEXU); F. W. Gould 210 (ENCB); G. B. Hinton 17232 (ENCB), 17414-bis (ENCB, MEXU); H. E. Iñiguez s.n. (ENCB); M. Mitastein 163 (ENCB).

Commelina diffusa Burm.f.: A. Espejo 5003 (UAMIZ), 6097 (UAMIZ); E. Matuda 21583 (MEXU); M. Mitastein 178 (ENCB).

Commelina erecta L.: A. Pulido 88 (UAMIZ).

Commelina leiocarpa Benth.: E. Lyonnet 2560 (MEXU); C. G. Pringle s.n. (MEXU); G. Serrano J. 53 (HUMO, UAMIZ).

Commelina orchioides Booth ex Lindl.: V. L. Cardoso 1268 (MEXU); A. Espejo 5684 (UAMIZ); D. B. Gold 181 (MEXU); R. Kral 25259 (ENCB); A. R. López-Ferrari 2229 (UAMIZ); J. Vázquez 2564 (MEXU).

Commelina tuberosa L.: G. Barroso 69 (UAMIZ); D. Engle 98 (MEXU); M. Flores C. 127 (UAMIZ); A. Kenton 24 (MEXU), 31 (MEXU); A. Pulido 66 (UAMIZ), 70 (UAMIZ).

*Gibasis karwinskyana (Schult. \& Schult.f.) Rohweder: A. R. López-Ferrari 2044 (UAMIZ).

*Gibasis linearis (Benth.) Rohweder: G. Barroso 64 (UAMIZ); A. Espejo 3642 (UAMIZ); A. R. López-Ferrari 1215 (UAMIZ); J. Vázquez 2241 (MEXU).

Gibasis pulchella (Kunth) Raf.: F. E. Allison 69 (MEXU); V. L. Cardoso 1373 (MEXU); I. Díaz V. 1046 (MEXU); A. Espejo 5708 (UAMIZ); D. B. Gold 362 (MEXU); D. R. Hunt 8002 (MEXU); A. R. López-Ferrari 2562 (UAMIZ); M. Mitastein 215 (ENCB); V. P. Mitchell 36 (MEXU); J. D. Traylor 4 (MEXU); J. Vázquez 2027 (MEXU), 2249 (ENCB).

* Gibasoides laxiflora (C.B. Clarke) D.R. Hunt: A. Espejo 3339 (UAMIZ).

*Thyrsanthemum macrophyllum (Greenm.) Rohweder: M. Flores C. 444 (UAMIZ); G. Serrano J. 54 (UAMIZ).

Tinantia erecta (Jacq.) Schltdl.: F. E. Allison 66 (MEXU); R. Cerros 226 (UAMIZ); I. Díaz V. 1133 (MEXU); J. M. Díaz 142 (ENCB); A. Espejo 3328 (UAMIZ), 5184 (UAMIZ), 6096 (UAMIZ); J. Espinosa 344 (ENCB, MEXU); E. L. Estrada 1694 (UAMIZ); M. Flores C. 282 (UAMIZ); D. B. Gold 231-A (MEXU); E. Matuda 25573 (MEXU); D. Ramírez-Cantú 219 (MEXU); G. Serrano J. 69 (UAMIZ).

Tradescantia commelinoides Schult. \& Schult.f.: E. Allison 89 (MEXU); F. A. Barkley 7408 (MEXU); L. Cabrera 404 (MEXU); L. B. Cole 2 (MEXU); A. Espejo 3346 (UAMIZ), 3636 (UAMIZ), 5709 (UAMIZ); J. Espinosa 90 (MEXU); M. Flores C. 129 (HUMO, UAMIZ); D. B. Gold 185 (MEXU); F. W. Gould 170 (ENCB, MEXU); A. Kenton 28 (MEXU); A. R. López-Ferrari 2216 (UAMIZ); M. Martínez 6 (ENCB); E. Matuda 25638 (MEXU); R. F. 
McAdams 61 (MEXU); F. Miranda 515 (MEXU); H. G. Quiram 39 (MEXU); J. D. Taylor 5 (MEXU); J. G. Teer 31 (MEXU); J. Vázquez 2028 (MEXU), 2250 (MEXU); F. W. Wyatt 50 (MEXU).

*Tradescantia crassifolia Cav. ssp. acaulis (M. Martens \& Galeotti) C.B. Clarke: E. Matuda 25624 (MEXU); F. Miranda 535 (MEXU).

*Tradescantia tepoxtlana Matuda: L. W. Boege 1982 (MEXU); R. Cerros 154 (UAMIZ); A. R. López-Ferrari 2376 (UAMIZ); M. Mitastein 197 (ENCB); C. G. Pringle 8463 (MEXU).

*Tripogandra amplexans Handlos: A. Espejo 3335 (UAMIZ); G. Serrano J. 75 (UAMIZ).

Tripogandra amplexicaulis (Klotzsch ex C.B. Clarke) Woodson: R. Cerros 59 (UAMIZ); A. Espejo 3337 (UAMIZ); J. Espinosa s.n. (ENCB); M. Mitastein 231 (ENCB); L. Paray 1733 (ENCB).

Tripogandra disgrega (Kunth) Woodson: J. Arvizu 93 (ENCB); J. M. Díaz 148 (ENCB); L. Paray 2172 (ENCB).

Tripogandra purpurascens (S. Schauer) Handlos: V. L. Cardoso 1366 (MEXU); R. Cerros 231 (UAMIZ); J. Vázquez s.n. (MEXU).

Weldenia candida Schult.f.: J. Arvizu 12 (MEXU).

\section{CYPERACEAE}

Bulbostylis tenuifolia (Rudge) J.F. MacBr.: E. Lyonnet 1503 (MEXU).

Carex hermannii Cochrane: J. Bonilla 1565 (HUMO, MEXU).

***Carex interjecta Reznicek: J. Freudenstein 2178 (MICH).

Carex longicaulis Boeck.: J. Vázquez 3330 (MEXU).

Carex lurida Wahlenb.: J. Bonilla 1533 (HUMO, MEXU).

Carex madrensis L.H. Bailey: E. Lyonnet 1860 (MEXU); C. G. Pringle 8260, s.n. (MEXU).

Carex psilocarpa Steud.: J. Bonilla 497 (HUMO); E. Lyonnet 2519 (ENCB, MEXU).

Cyperus cuspidatus Kunth: E. L. Estrada 1326 (MEXU).

Cyperus esculentus L.: L. W. Boege 1919 (MEXU); J. Espinosa 36 (ENCB); E. L. Estrada 1657 (MEXU).

Cyperus hermaphroditus (Jacq.) Standl.: V. Benhumea S. 317 (ENCB, MEXU); J. Bonilla 1112 (HUMO, MEXU); I. Díaz V. 1132 (MEXU); R. Díaz 32 (HUMO); D. Engle 102 (MEXU); J. Espinosa 35 (MEXU); M. Gutiérrez 404 (MEXU); E. Lyonnet 1819 (ENCB, MEXU); A. Pérez J. 508 (MEXU); R. E. Rodríguez 667 (MEXU); J. Villanueva O. 6 (HUMO).

Cyperus lanceolatus Poir.: J. Bonilla 163 (HUMO); E. Matuda 25605 (MEXU).

Cyperus manimae Kunth: R. Cerros 2 (UAMIZ); F. Gallegos H. 521 (MEXU); E. Lyonnet 2409 (MEXU).

Cyperus mutisii (Kunth) Griseb.: L. W. Boege 1920 (MEXU); E. L. Estrada 1122 (MEXU, UAMIZ); E. Lyonnet 718 (MEXU); A. Pulido 11 (UAMIZ).

Cyperus niger Ruiz \& Pav.: J. Bonilla 508 (HUMO), 1521 (HUMO), 1550 (HUMO), 1558 (HUMO); E. Lyonnet 2433 (MEXU); E. Matuda 25598 (MEXU).

Cyperus ochraceus Vahl: A. Pulido 22 (UAMIZ).

Cyperus seslerioides Kunth: J. E. Allison 100 (MEXU); J. Espinosa 15 (MEXU); A. Pulido 60 (UAMIZ); L. I. Nevling 338 (MEXU); J. Vasquez 1881 (MEXU). 
Cyperus spectabilis Link: E. Lyonnet 717 (MEXU).

Cyperus tenerrimus J. Presl \& C. Presl: E. L. Estrada 115 (MEXU).

Eleocharis acicularis (L.) Roem. \& Schult.: J. Bonilla 424 (HUMO), 457 (HUMO), 1514

(HUMO), 1530 (HUMO), 1594 (HUMO); E. Lyonnet 2516 (MEXU); E. Matuda 25603 (MEXU); F. Miranda 187 (MEXU).

Eleocharis bonariensis Nees: J. Bonilla 484 (HUMO, MEXU).

Eleocharis densa Benth.: J. Bonilla 360 (MEXU).

Eleocharis dombeyana Kunth: R. Cruz 527 (ENCB, MEXU).

Eleocharis filiculmis Kunth: J. Bonilla 484 (HUMO, MEXU).

Eleocharis macrostachya Britton: J. Bonilla 1549 (HUMO), 1538-A (HUMO); E. Gallegos 18 (MEXU); P. R. Matosic 1284 (MEXU).

Eleocharis montevidensis Kunth: J. Bonilla 166 (HUMO); V. L. Cardoso 1543 (HUMO, MEXU, UAMIZ); E. Matuda 25944 (MEXU).

Karinia mexicana (C.B. Clarke ex Britton) Reznicek \& McVaugh: A. Espejo 5701 (UAMIZ).

Kyllinga odorata Vahl: R. Cerros 139 (UAMIZ); L. B. Cole 41 (MEXU).

Scirpus californicus (C.A. Mey.) Steud.: J. Bonilla 47 (HUMO), 156 (HUMO), 346 (HUMO), 357 (HUMO), 1531 (HUMO); V. L. Cardoso 1183 (HUMO); R. Galván 710-A (ENCB, MEXU); M. González G. 26 (MEXU).

\section{DIOSCOREACEAE}

Dioscorea convolvulacea Schltdl. \& Cham. var. convolvulacea: E. Matuda 30149 (MEXU), 37317 (MEXU); C. G. Pringle s.n. (MEXU); A. Pulido 78 (UAMIZ); R. Ramírez R. 259 (HUMO).

Dioscorea convolvulacea Schltdl. \& Cham. var. grandifolia (Schltdl.) Uline ex R. Knuth: $R$. Cerros 217 (UAMIZ); A. R. López-Ferrari 2031-bis (UAMIZ).

*Dioscorea galeottiana Kunth: G. Barroso 56 (MEXU, UAMIZ); R. Cerros 256 (HUMO); J. Espinosa s.n. (MEXU); G. Flores 149 (MEXU); G. B. Hinton 17419-bis (ENCB, MEXU); E. Lyonnet 2555 (MEXU); E. Matuda 21629 (MEXU), 30141 (ENCB), 30143 (MEXU), 30152 (ENCB); M. Mitastein 158 (ENCB); R. Ramírez R. 263 (HUMO), 269 (HUMO, MEXU).

**Dioscorea gallegosi Matuda: R. Cerros 75 (UAMIZ).

Dioscorea liebmannii Uline: E. Lyonnet 2985 (MEXU).

Dioscorea lobata Uline: J. Bonilla 1124 (HUMO, MEXU); E. Lyonnet 650 (MEXU).

*Dioscorea morelosana (Uline) Matuda: B. P. Reko s.n. (MEXU); J. Vázquez 3818 (MEXU).

Dioscorea nelsonii Uline ex R. Knuth: R. Medina 91 (MEXU).

**Dioscorea pumicicola Uline: C. G. Pringle 7227 (MEXU).

*Dioscorea remotiflora Kunth: I. De la Cruz 1421 (HUMO, MEXU); O. Dorado R. 1430 (HUMO); E. Matuda 30137 (ENCB), 30145 (ENCB), 30147 (ENCB); C. G. Pringle s.n. (ENCB, MEXU); B. P. Reko s.n. (MEXU).

*Dioscorea subtomentosa Miranda: R. E. Rodríguez 1006 (MEXU).

*Dioscorea ulinei Greenm. ex R. Knuth: J. Espinosa 352 (ENCB, MEXU); E. Matuda 32635 (MEXU). 
*Dioscorea urceolata Uline: G. Barroso 14 (UAMIZ), 55 (UAMIZ), 72 (UAMIZ); $R$. Cerros 47 (UAMIZ), 219 (UAMIZ); A. Espejo 4278 (UAMIZ); J. Espinosa 34 (ENCB, MEXU), 206 (MEXU), 286 (ENCB); M. L. Flores 18 (UAMIZ); M. T. Germán 570 (ENCB, MEXU); D. B. Gold 249 (ENCB, MEXU); G. B. Hinton 17406-bis (ENCB, MEXU); E. Matuda 30138 (MEXU), 30142 (MEXU), 30146 (MEXU), 30150 (MEXU); C. G. Pringle s.n. (MEXU); D. Ramírez-Cantú 214 (MEXU); R. Ramírez R. 430 (HUMO, MEXU).

\section{ERIOCAULACEAE}

*Eriocaulon benthamii Kunth: A. Espejo 4025 (UAMIZ); S. D. Koch 832 (MEXU); E. Matuda s.n. (MEXU).

Eriocaulon ehrenbergianum Klotzsch ex Körn: J. Bonilla 1515 (HUMO, MEXU), 1481 (HUMO, MEXU).

Eriocaulon microcephalum Kunth: J. Bonilla 1501 (HUMO, UAMIZ).

\section{HYDROCHARITACEAE}

Egeria densa Planch.: J. Bonilla 270 (HUMO, MEXU), 281 (HUMO), 526 (HUMO, MEXU), 1556, (HUMO, MEXU); L. Cabrera R. 403 (MEXU); V. L. Cardoso 129 (HUMO), 1454 (HUMO); P. Durán 4 (UAMIZ); A. Espejo 3475 (UAMIZ); S. Zamudio 10241 (ENCB, HUMO, MEXU).

\section{HYPOXIDACEAE}

Hypoxis decumbens L.: A. Espejo 3629 (MEXU, UAMIZ), 4986 (MEXU, UAMIZ).

Hypoxis mexicana Schult. \& Schult.f.: G. Barroso 51 (UAMIZ); A. Bonfil C. 1010 (MEXU); J. Bonilla 1110 (HUMO, MEXU); G. Manzanero 1182 (MEXU); A. Pulido 41 (UAMIZ), 57 (UAMIZ), 59 (UAMIZ), 68 (UAMIZ); H. G. Quiram 15 (MEXU); R. Torres C. 14010 (MEXU).

Hypoxis potosina Brackett: A. Espejo 4990 (UAMIZ), 5638 (UAMIZ); A. Pulido 16 (UAMIZ), 24 (UAMIZ); R. Girón T. s.n. (UAMIZ).

*Hypoxis tepicensis Brackett: R. Cerros s.n. (UAMIZ); A. Espejo 5911 (UAMIZ).

\section{IRIDACEAE}

Nemastylis tenuis (Herb.) Baker var. tenuis: R. Cerros 57 (UAMIZ).

*Sisyrinchium angustissimum (B.L. Rob. \& Greenm.) Greenm. \& C.H. Thomps.: J. L. Brunhuber 11 (UAMIZ); S. Cacho s.n. (UAMIZ); A. Espejo 6098 (UAMIZ); E. L. Estrada 1822 (UAMIZ); A. R. López-Ferrari 2382 (UAMIZ); E. Lyonnet 1816 (MEXU); F. Miranda 3767 (MEXU); G. Serrano J. 27 (UAMIZ).

Sisyrinchium cernuum (E.P. Bicknell) Kearney: A. Espejo 3497 (UAMIZ).

*Sisyrinchium macrophyllum Greenm.: A. Pulido 7 (UAMIZ), 10 (UAMIZ).

Sisyrinchium scabrum Schltdl. \& Cham.: F. E. Allison 71 (MEXU); J. Bonilla 1611 (HUMO, UAMIZ); C. E. Boyd 100 (MEXU); J. Ceja 96 (UAMIZ); V. L. Cardoso 1286 (MEXU); $A$. Espejo 1000 (UAMIZ), 3313 (UAMIZ); A. Kenton 36 (MEXU); E. Lyonnet 214 (MEXU); A. Pulido 45 (UAMIZ); H. G. Quiram 23 (MEXU); W. T. Smith 50 (MEXU); M. Ulloa s.n. (MEXU); J. Vázquez 2616 (MEXU).

*Sisyrinchium schaffneri S. Watson: E. Rojo G. 2 (UAMIZ). 
Sisyrinchium tenuifolium Humb. \& Bonpl. ex Willd.: J. Ceja 95 (UAMIZ); A. Espejo 5002 (UAMIZ), 5410 (UAMIZ), 5427 (UAMIZ), 5702 (UAMIZ); M. Flores C. 452 (UAMIZ); C. G. Pringle 11863 (UAMIZ); A. Pulido 83 (UAMIZ).

*Sisyrinchium tolucense Peyr.: J. Bonilla 1551 (HUMO, MEXU, UAMIZ); A. Espejo 4585 (UAMIZ); A. R. López-Ferrari 763 (UAMIZ), 2563 (UAMIZ); H. Pedraza s.n. (ENCB).

**Tigridia matudae Molseed: A. Rodríguez C. 2938 (UAMIZ).

Tigridia meleagris (Lindl.) G. Nicholson: R. Cerros 309 (UAMIZ); A. Espejo 2557 (UAMIZ), 5434 (UAMIZ); A. R. López-Ferrari 1232 (UAMIZ).

*Tigridia multiflora (Herb.) Ravenna: A. Espejo 3849 (MEXU, UAMIZ); G. B. Hinton 17231-bis (ENCB); A. R. López-Ferrari 2031 (UAMIZ).

***Tigridia tepoxtlana Ravenna: A. Espejo 2043 (MEXU, UAMIZ), 4274 (UAMIZ).

\section{JUNCACEAE}

Juncus arcticus Willd. var. andicola (Hook.) Balslev: V. L. Cardoso 1362 (UAMIZ); R. Galván 710-b (ENCB, MEXU, UAMIZ).

Juncus arcticus Willd. var. mexicanus (Willd. ex Schult. \& Schult.f.) Balslev: J. Bonilla 422 (HUMO, MEXU); D. Martínez A. s.n. (MEXU); A. Pulido 37 (UAMIZ), 43 (UAMIZ). Juncus arcticus Willd. var. montanus (Engelm.) Balslev: V. L. Cardoso 1345 (MEXU). Juncus ebracteatus Liebm.: J. Bonilla 347, 446, 487, 546, 1538 (HUMO, MEXU).

Juncus liebmannii J.F. MacBr. var. liebmannii: E. Lyonnet 2483 (MEXU); W. T. Smith 29 (MEXU); F. W. Wyatt 79 (MEXU).

Luzula caricina E. Mey.: O. C. Bohillos 43 (MEXU); V. L. Cardoso 1301 (MEXU); A. Pulido 49 (UAMIZ), 53 (UAMIZ).

Luzula racemosa Desv.:V. L. Cardoso 1031 (HUMO, UAMIZ).

JUNCAGINACEAE

Lilaea scilloides (Poir.) Hauman: J. Bonilla 442 (HUMO), 1513 (HUMO), 1541 (HUMO).

\section{LEMNACEAE}

Lemna aequinoctialis Welw.: J. Bonilla 237 (MEXU), 376 (MEXU), 430 (MEXU), 485 (MEXU), 598 (MEXU), 672 (HUMO), 1528 (MEXU), 1557 (HUMO, MEXU, UAMIZ); N. C. Fassett 28444 (MEXU), 28457 (MEXU).

Lemna gibba L.: J. Bonilla 168 (HUMO, MEXU), 344 (HUMO, MEXU), 597 (HUMO, MEXU).

\section{MELANTHIACEAE}

Schoenocaulon officinale (Schltdl. \& Cham.) A. Gray ex Benth.: E. Lyonnet 521200019 (MEXU).

**Schoenocaulon pringlei Greenm.: A. R. López-Ferrari 2230 (UAMIZ); S. Zamudio 9237 (MEXU, UAMIZ).

***Schoenocaulon tenue Brinker: G. Barroso 63 (UAMIZ); A. R. López-Ferrari 2039-b (UAMIZ); D. Martínez A. s.n. (MEXU).

*Stenanthium frigidum (Schltdl. \& Cham.) Kunth: H. H. Iltis 254 (MEXU); L. Torres 1069 (MEXU); J. Vázquez 1914 (MEXU). 


\section{ORCHIDACEAE}

Barkeria obovata (C. Presl) Christenson: A. Espejo 5937 (UAMIZ).

Bletia adenocarpa Rchb.f.: A. Espejo 5690 (UAMIZ).

*Bletia campanulata Lex.: R. Cerros 1833 (UAMIZ); A. Espejo 2621 (UAMIZ), 5700

(UAMIZ); A. Flores C. 284 (HUMO), 919 (HUMO).

*Bletia coccinea Lex.: R. Cerros 1832 (UAMIZ); A. Espejo 5436 (UAMIZ).

Bletia gracilis G. Lodd.: R. Cerros 201 (UAMIZ); A. Espejo 5696 (UAMIZ); A. R. LópezFerrari 738 (UAMIZ).

Bletia lilacina A. Rich. \& Galeotti: A. Espejo 5932 (UAMIZ).

Bletia macristhmochila Greenm.: A. Espejo 5686 (UAMIZ).

Bletia neglecta Sosa: A. Espejo 5610 (UAMIZ); J. García-Cruz 948 (UAMIZ); A. R. LópezFerrari 2413 (UAMIZ), 2512 (UAMIZ); B. Pérez 1018(UAMIZ); G. Sánchez s.n. (UAMIZ);

L. Sánchez S. 390 (UAMIZ); V. Sánchez 6 (UAMIZ); J. Vázquez 4958 (MEXU).

Bletia parkinsonii Hook.: O. Nagel \& J. González 3671 (AMES).

*Bletia punctata Lex.: A. Espejo 3667 (UAMIZ).

Bletia purpurata A. Rich. \& Galeotti: R. Cerros 224 (UAMIZ), A. Espejo 2665 (UAMIZ), 2752 (UAMIZ), 3844 (UAMIZ), 5425 (UAMIZ), 5900 (UAMIZ); J. García-Cruz 902 (UAMIZ), 904 (UAMIZ); A. R. López-Ferrari 2039 (UAMIZ), 2524 (UAMIZ); R. Jiménez M. 2012 (UAMIZ), 2167 (UAMIZ).

Bletia roezlii Rchb.f.: A. R. López-Ferrari 2542 (UAMIZ).

Brachystele affinis (C. Schweinf.) Burns-Bal. \& R. González: B. Cruz sub E. Oestlund 2160 (MEXU).

Bulbophyllum nagelii L.O. Williams: H. D. Sawyer 499 (MEXU).

Cattleya aurantiaca (Bateman ex Lindl.) P.N. Don: E. L. Estrada 868 (MEXU); R. Jiménez M. 2018 (UAMIZ).

Clowesia thylaciochila (Lem.) Dodson: A. Flores C. 240 (UAMIZ).

*Corallorhiza bulbosa A. Rich. \& Galeotti: J. García-Cruz 771 (UAMIZ); A. R. LópezFerrari 754 (UAMIZ), 2547 (UAMIZ).

*Corallorhiza ehrenbergii Rchb.f.: R. Jiménez M. 2007 (UAMIZ).

Corallorhiza maculata (Raf.) Raf.: A. Espejo 5703 (UAMIZ), 5715 (UAMIZ); R. Jiménez M. 1948 (UAMIZ); A. R. López-Ferrari 755 (UAMIZ), 762 (UAMIZ), 2215 (UAMIZ); L. Sánchez S. 528 (UAMIZ).

Corallorhiza odonthorhiza (Willd.) Nutt.: A. Espejo 5931 (UAMIZ).

Corallorhiza williamsii Correll: O. Nagel \& J. González sub E. Oestlund 6608 (AMES).

Cranichis subumbellata A. Rich. \& Galeotti: E. Lyonnet 1356 (MEXU).

Cyclopogon saccatus (A. Rich. \& Galeotti) Schltr.: E. Lyonnet 530600018 (MEXU).

Cypripedium irapeanum Lex.: J. Vázquez 4000 (MEXU); O. Nagel \& J. González sub E. Oestlund 1302 (AMES).

Deiregyne pyramidalis (Lindl.) Burns-Bal.: A. Espejo 2670 (UAMIZ), 3460 (UAMIZ), 3586 (UAMIZ), 5393 (UAMIZ), 5426 (UAMIZ), 5622 (UAMIZ), 5641 (UAMIZ), 5952 (UAMIZ); A. Flores C. 1071 (UAMIZ); J. García-Cruz 782 (UAMIZ); A. R. López-Ferrari 2450 (UAMIZ), 2452 (UAMIZ), 2584 (UAMIZ); L. Sánchez S. 466 (UAMIZ).

*Deiregyne rhombilabia Garay: A. Espejo 5944 (UAMIZ), 5953 (UAMIZ); J. García-Cruz 783 (UAMIZ). 
*Deiregyne tenuiflora (Greenm.) Burns-Bal.: A. Espejo 3167 (UAMIZ), 5938 (UAMIZ); C. G. Pringle 6995 (MEXU).

*Dichaea squarrosa Lindl.: A. Espejo 2727 (UAMIZ), 2742 (UAMIZ), 5389 (UAMIZ); $A$. Flores C. 321 (HUMO); L. Sánchez S. 347 (UAMIZ).

Dichromanthus cinnabarinus (Lex.) Garay: R. Cerros 234 (UAMIZ); A. Espejo 3846 (UAMIZ), 5922 (UAMIZ).

Encyclia microbulbon (Hook.) Schltr.: A. Espejo 2632 (UAMIZ).

*Encyclia spatella (Rchb.f.) Schltr.: A. Flores C. 641 (UAMIZ).

Epidendrum anisatum Lex.: A. Espejo 2684 (UAMIZ), 3485 (UAMIZ), 3560 (UAMIZ), 5635 (UAMIZ); A. R. López-Ferrari 2518 (UAMIZ).

Epidendrum eximium L.O. Williams: L. Sánchez S. 393 (AMO, UAMIZ)

*Epidendrum matudae L.O. Williams: A. Espejo 2038 (UAMIZ), 3430 (UAMIZ), 3585 (UAMIZ); J. García Cruz 583 (UAMIZ).

*Epidendrum miserum Lindl.: A. R. López-Ferrari 2405 (UAMIZ).

Epidendrum parkinsonianum Hook.: O. Nagel sub. E. Oestlund 2876 (AMES); A. Flores C. s.n. (UAMIZ); A. R. López-Ferrari 2868 (UAMIZ); E. Lyonnet 550400019 (MEXU).

Galeottiella sarcoglossa (A. Rich. \& Galeotti) Schltr.: J. García-Cruz 772 (AMO, UAMIZ); A. R. López-Ferrari 2552 (UAMIZ); J. Rzedowski 21625 (ENCB).

Goodyera striata Rchb.f.: A. Espejo 5586 (UAMIZ); J. García-Cruz 917 (UAMIZ).

Govenia liliacea (Lex.) Lindl.: L. B. Cole 31 (MEXU); A. Espejo 3283 (UAMIZ); A. Flores C. 7108 (UAMIZ); A. R. López-Ferrari 1236 (UAMIZ); E. Lyonnet 881 (MEXU); R. F. McAdams 66 (MEXU); A. Pulido 47 (UAMIZ), 55 (UAMIZ), 63 (UAMIZ); L. Sánchez S. 517 (UAMIZ).

Govenia superba (Lex.) Lodd.: A. Espejo 861 (UAMIZ), 3297 (UAMIZ); A. R. López-Ferrari 2219 (UAMIZ); C. L. Lundell \& A. A. Lundell 12327 (MICH); A. Pulido 73 (UAMIZ); L. Sánchez S. 510 (AMO, UAMIZ).

*Greenwoodia sawyeri (Standl. \& L.O. Williams) Burns-Bal.: A. Espejo 4275 (UAMIZ); J. García-Cruz 950 (AMO, UAMIZ); A. R. López-Ferrari 2370 (UAMIZ), 2514 (UAMIZ); E. Lyonnet 510900035 (MEXU).

Habenaria alata Hook.: J. González \& O. Nagel sub E. Oestlund 1207 (F).

*Habenaria calicis R. González: J. García-Cruz 776 (UAMIZ).

Habenaria crassicornis Lindl.: G. Barroso 59 (UAMIZ); J. L. Brunhuber 13 (UAMIZ); A. Espejo 5568, (UAMIZ); A. Flores C. 317 (HUMO), 318 (HUMO), 435 (HUMO); A. R. López-Ferrari 2032 (UAMIZ).

Habenaria entomantha (Lex.) Lindl.: A. Espejo 5699 (UAMIZ); M. Flores C. 119 (UAMIZ); A. R. López-Ferrari 2228 (UAMIZ).

Habenaria filifera S. Watson: J. González sub E. Oestlund 1216 (MEXU); 3029 (MEXU).

Habenaria flexuosa Lindl.: A. Espejo 870 (UAMIZ).

Habenaria guadalajarana S. Watson: R. Jiménez M. 2171 (UAMIZ).

Habenaria novemfida Lindl.: J. Ceja 75 (UAMIZ); R. Cerros 227 (UAMIZ); A. Espejo 2570 (UAMIZ), 3845 (UAMIZ), 5885 (UAMIZ); A. R. López-Ferrari 364 (UAMIZ); E. Lyonnet 500800007 (MEXU).

Habenaria oreophila Greenm.: E. Lyonnet 510900037 (MEXU). 
*Habenaria rosulifolia Espejo \& López-Ferrari: A. Espejo 885 (UAMIZ); A. R. LópezFerrari 2554 (UAMIZ), 2558 (UAMIZ).

Habenaria strictissima Rchb.f.: E. Lyonnet 56080009 (MEXU).

Habenaria uncata R. Jiménez, L. Sánchez \& García-Cruz: E. Lyonnet 560800010 (MEXU).

Habenaria virens A. Rich. \& Galeotti: A. R. López-Ferrari 2531 (UAMIZ).

Hexalectris grandiflora (A. Rich. \& Galeotti) L.O. Williams: J. García-Cruz 769 (UAMIZ).

*Hintonella mexicana Ames: A. Espejo 2682 (UAMIZ), 5419 (UAMIZ); J. García-Cruz 688 (UAMIZ).

*Isochilus bracteatus (Lex.) Salazar \& Soto Arenas: A. Flores C. 640 (UAMIZ); A. R. López-Ferrari 2726 (UAMIZ); I. Luna 470 (MEXU).

*Laelia autumnalis (Lex.) Lindl.: R. Cerros 280 (HUMO); A. R. López-Ferrari 2409 (UAMIZ); L. Sánchez S. 387 (AMO, UAMIZ).

Leochilus carinatus (Knowles \& Westc.) Lindl.: A. Espejo 2584 (UAMIZ).

*Lepanthes nagelii Salazar \& Soto Arenas: A. Espejo 3292 (UAMIZ), 5612 (UAMIZ); A. Flores C. 400 (UAMIZ); A. R. López-Ferrari 2180 (UAMIZ); L. Sánchez S. 345 (UAMIZ).

Liparis cordiformis C. Schweinf.: A. Espejo 5898 (UAMIZ).

*Liparis greenwoodiana Espejo; A. Espejo 2596 (AMO, UAMIZ), 5431 (UAMIZ), 5888 (UAMIZ); A. Flores C. 243 (HUMO), 251 (HUMO), 267 (UAMIZ); A. R. López-Ferrari 1227 (UAMIZ), 2369 (UAMIZ); L. Sánchez S. 531 (UAMIZ).

Liparis vexillifera (Lex.) Cogn.: A. Espejo 2623 (UAMIZ); A. R. López-Ferrari 1233 (UAMIZ), 2511 (UAMIZ).

Malaxis abieticola Salazar \& Soto-Arenas: A. Espejo 5587 (UAMIZ); R. Jiménez M. 921 (UAMIZ); G. Salazar 3388 (AMO).

*Malaxis alvaroi García-Cruz, R. Jiménez \& L. Sánchez: A. Espejo 5430 (AMO, UAMIZ). Malaxis brachyrrhynchos (Rchb.f.) Ames: A. Espejo 860 (UAMIZ), 2586 (UAMIZ), 2610 (UAMIZ), 2619 (UAMIZ), 2636 (UAMIZ), 3285 (UAMIZ), 3322 (UAMIZ), 3622 (UAMIZ), 3627 (UAMIZ), 5429 (UAMIZ), 5437 (UAMIZ), 5685 (UAMIZ); M. Flores $C$. 451 (UAMIZ); A. R. López-Ferrari 746 (UAMIZ), 1223 (UAMIZ), 2218 (UAMIZ), 2410 (UAMIZ); A. Pulido 74 (UAMIZ), 61-bis (UAMIZ); L. Sánchez S. 490 (UAMIZ), 503 (UAMIZ), 515 (UAMIZ).

Malaxis brachystachya (Rchb.f.) Kuntze: A. Espejo 5437 (UAMIZ).

Malaxis carnosa (Kunth) C. Schweinf.: A. Flores C. 906 (UAMIZ).

Malaxis ehrenbergii (Rchb.f.) Kuntze: J. Bonilla 751 (HUMO); L. Sánchez 534 (AMO).

Malaxis fastigiata (Rchb.f.) Kuntze: A. De Nova s.n. (UAMIZ); A. Espejo 5577 (UAMIZ); A. Pulido 54 (UAMIZ), 61 (UAMIZ), 77 (UAMIZ).

Malaxis lepidota (Finet) Ames: J. Ceja 76(UAMIZ); Espejo 2587 (UAMIZ), 3459 (UAMIZ), 5440 (UAMIZ).

Malaxis maianthemifolia Schltdl. \& Cham.: A. Espejo 2678 (UAMIZ), 3280 (UAMIZ), 5402 (UAMIZ), 5691 (UAMIZ); J. García-Cruz 907 (UAMIZ); A. R. López-Ferrari 2179 (UAMIZ). 
*Malaxis myurus (Lindl.) Kuntze: T. Chew s.n. (UAMIZ); A. Espejo 5432 (UAMIZ); J. García-Cruz 859 (UAMIZ); A. R. López-Ferrari 2225 (UAMIZ).

***Malaxis palustris Espejo \& López-Ferrari: J. García-Cruz 751 (AMO, UAMIZ).

**Malaxis ribana Espejo \& López-Ferrari: E. Lyonnet 530600010 (MEXU), 550800034 (MEXU), 560800014 (MEXU); H. D. Sawyer 982 (F).

*Malaxis rosei Ames: R. Jiménez M. 2006 (UAMIZ); A. R. López-Ferrari 2548 (UAMIZ); E. Lyonnet 617 (MEXU); A. Pulido 74-bis (UAMIZ).

*Malaxis rosilloi R. González \& E.W. Greenw.: R. Cerros 310 (HUMO, UAMIZ); A. Espejo 2582 (UAMIZ), 2635 (UAMIZ), 3288 (UAMIZ), 3343 (UAMIZ), 3633 (UAMIZ), 5415 (UAMIZ), 5688 (UAMIZ); J. García-Cruz 867 (UAMIZ); A. R. López-Ferrari 1229 (UAMIZ).

Malaxis salazarii Catling: A. Espejo 881 (UAMIZ), 3315 (UAMIZ), 5697 (UAMIZ), 5711 (UAMIZ); R. Jiménez M. 1985 (UAMIZ); A. R. López-Ferrari 2223 (UAMIZ), 2543 (UAMIZ).

Malaxis soulei L.O. Williams: A. Flores C. 915 (UAMIZ), 916 (UAMIZ); L. Sánchez S. 535 (AMO, UAMIZ).

Malaxis streptopetala (B.L. Rob. \& Greenm.) Ames: A. Espejo 884 (UAMIZ), 3316 (UAMIZ), 5710 (UAMIZ); A. R. López-Ferrari 2224 (UAMIZ).

Malaxis stricta L.O. Williams: J. González sub E. Oestlund 1561 (AMES).

Malaxis unifolia Michx.: A. R. López-Ferrari 2540 (UAMIZ).

Maxillaria lexarzana Soto-Arenas \& Chiang: J. González sub E. Oestlund 604 (MEXU).

*Microthelys minutiflora (A. Rich. \& Galeotti) Garay: A. Espejo 1001 (UAMIZ), 3461 (UAMIZ), 3492 (UAMIZ), 5928 (UAMIZ), 5930 (UAMIZ), 5948 (UAMIZ), 5963 (UAMIZ).

*Microthelys nutantiflora (Schltr.) Garay: A. Espejo 5949 (UAMIZ); J. García-Cruz 757 (UAMIZ).

Oncidium brachyandrum Lind1.: R. Jiménez M. 912 (AMO).

Oncidium cebolleta (Jacq.) Sw.: J. Vázquez C. 3978 (MEXU); R. Medina 81 (UAMIZ).

*Oncidium geertianum C. Morren: A. Espejo 3432 (UAMIZ).

Oncidium graminifolium (Lind.) Lindl.: E. Hágsater 3689 (AMO).

Oncidium microstigma Rchb.f.: A. Espejo 2601 (UAMIZ); R. Jiménez M. 902 (UAMIZ); J. Vázquez 3561 (MEXU).

Oncidium pachyphyllum Hook.: M. Flores C. 10 (UAMIZ).

Oncidium reichenheimii (Linden \& Rchb.f.) Garay \& Stacy: A. Espejo \& A. Flores C. 2738 (UAMIZ).

Oncidium unguiculatum Lindl.: A. Espejo 2686 (UAMIZ), 3559 (UAMIZ); R. Jiménez M. 1867 (UAMIZ); E. Rojo G. 5 (UAMIZ).

Platanthera brevifolia (Greene) Kraenzl.: A. Espejo 5580 (UAMIZ); A. R. López-Ferrari 2549 (UAMIZ); L. Sánchez S. 485 (UAMIZ).

*Platanthera volcanica Lindl.: A. Espejo 3882 (UAMIZ); A. R. López-Ferrari 370 (UAMIZ); E. Lyonnet 40900025 (MEXU).

***Pleurothallis nigriflora L.O. Williams: A. Espejo 3431 (UAMIZ); J. García-Cruz 954 (UAMIZ). 
Pleurothallis oestlundiana L.O. Williams: A. Espejo 5396 (UAMIZ).

Pleurothallis retusa (Lex.) Lindl.: J. L. Brunhuber 21 (UAMIZ); A. Espejo 722 (UAMIZ), 2748 (UAMIZ), 5404 (UAMIZ), 5634 (UAMIZ), 6026 (UAMIZ); E. Rojo G. 9 (UAMIZ); V. Sánchez 4 (UAMIZ).

***Ponera dressleriana Soto Arenas: A. Espejo 5962 (UAMIZ); J. Vázquez 3506 (MEXU). Ponthieva brenesii Lindl.: E. Lyonnet 41200085 (MEXU).

Ponthieva ephippium Rchb.f.: A. Espejo 2683 (UAMIZ), 5899 (UAMIZ); A. Flores C. 448 (HUMO).

Ponthieva hildae R. González \& R. Soltero: E. Lyonnet 500800055 (MEXU).

Ponthieva racemosa (Walter) C. Mohr: A. Flores C. 449 (HUMO).

Ponthieva schaffneri (Rchb.f.) E.W. Greenw.: E. Lyonnet 520900024 (MEXU), 521100039 (MEXU).

Prescottia tubulosa (Lindl.) L.O. Williams: A. Espejo 3491 (UAMIZ), 5926 (UAMIZ); $R$. Jiménez M. 2037 (UAMIZ); A. R. López-Ferrari 2434 (UAMIZ).

*Prosthechea linkiana (Klotzsch) W.E. Higgins: A. Espejo 774 (UAMIZ), 2556 (UAMIZ), 2578 (UAMIZ), 5956 (UAMIZ); A. Flores C. 229 (UAMIZ); M. Flores C. 89 (UAMIZ); J. García-Cruz 784 (UAMIZ); A. R. López-Ferrari 2175 (UAMIZ), 2448 (UAMIZ).

Prosthechea michuacana (Lex.) W.E. Higgins: J. González sub E. Oestlund 2776 (MEXU).

Prosthechea pringlei (Rolfe) W.E. Higgins: A. Espejo 5640 (UAMIZ).

Prosthechea rhombilabia (S. Rosillo) W.E. Higgins: A. Espejo 2751 UAMIZ), 5637 (UAMIZ); A. R. López-Ferrari 2372 (UAMIZ).

Prosthechea varicosa (Bateman ex Lindl.) W.E. Higgins: A. Espejo 3517 (UAMIZ); O. Nagel 2176 (MEXU); C. G. Pringle 6977 (MEXU).

*Rhynchostele aptera (Lex.) Soto Arenas \& Salazar: A. Espejo 3525 (UAMIZ), 5636 (UAMIZ), 5643 (UAMIZ).

*Rhynchostele cervantesii (Lex.) Soto Arenas \& Salazar: A. Espejo 649 (UAMIZ), 777 (UAMIZ), 2687 (UAMIZ), 3481 (UAMIZ), 3510 (UAMIZ), 5391 (UAMIZ), 5401 (UAMIZ), 6040 (UAMIZ); R. Jiménez M. 1848 (UAMIZ), 2033 (UAMIZ); A. R. LópezFerrari 2414 (UAMIZ); E. Rojo G. 4 (UAMIZ).

*Rhynchostele maculata (Lex.) Soto Arenas \& Salazar: A. Espejo 563 (UAMIZ); J. GarcíaCruz 952 (UAMIZ); A. R. López-Ferrari 2373 (UAMIZ).

Sarcoglottis assurgens Schltr.: J. González sub E. Oestlund 2145 (MEXU).

Sarcoglottis schaffneri (Rchb.f.) Ames: A. Espejo 805 (UAMIZ), 871 (UAMIZ), 2669 (UAMIZ), 2673 (UAMIZ), 2679 (UAMIZ), 2799 (UAMIZ), 5403 (UAMIZ); R. Jiménez M. 1872 (UAMIZ); A. R. López-Ferrari 2177 (UAMIZ), 2735 (UAMIZ).

*Schiedeella albovaginata (C. Schweinf.) Burns-Bal.: J. Ceja 799 (UAMIZ), 806 (UAMIZ); A. Espejo 2688 (UAMIZ), 2755 (UAMIZ); A. R. López-Ferrari 2587 (UAMIZ).

Schiedeella crenulata (L.O. Williams) Espejo \& López-Ferrari: A. R. López-Ferrari 2591 (UAMIZ).

*Schiedeella densiflora (C. Schweinf.) Burns-Bal.: J. Garcia-Cruz 513 (UAMIZ); E. Lyonnet 889 (MEXU); L. Sánchez S. 513 (UAMIZ).

Schiedeella eriophora (B.L. Rob. \& Greenm.) Schltr.: A. Espejo 5657 (UAMIZ); A. R. López-Ferrari 2053 (UAMIZ). 
Schiedeella garayana R. González: J. Ceja 798 (UAMIZ).

Schiedeella hyemalis (A. Rich. \& Galeotti) Burns-Bal.: A. Flores C. 329 (HUMO).

Schiedeella llaveana (Lindl.) Schltr. var. llaveana: A. Espejo 3588 (UAMIZ), 4018(UAMIZ), 5621 (UAMIZ), 5656 (UAMIZ); A. Flores C. 1038 (UAMIZ); M. Flores C. 407 (UAMIZ); A. R. López-Ferrari 2422 (UAMIZ).

Schiedeella sparsiflora (C. Schweinf.) Burns-Bal.: O. Nagel \& J. González sub. E. Oestlund 1554 (AMO).

*Spiranthes graminea Lindl.: J. Bonilla 490 (HUMO, MEXU); A. Espejo 5406 (UAMIZ); A. R. López-Ferrari 2227 (UAMIZ).

*Stanhopea hernandezii (Kunth) Schltr.: A. Espejo 2735 (UAMIZ), 3635 (UAMIZ), 5420 (UAMIZ); M. Flores C. 443 (HUMO, UAMIZ).

Stenorrhynchos aurantiacum (Lex.) Lindl.: G. Barroso 66 (UAMIZ); R. Cerros 212 (UAMIZ); A. Espejo 855 (UAMIZ), 859 (UAMIZ), 2559 (UAMIZ), 5423 (UAMIZ), 5704 (UAMIZ); J. García-Cruz 746 (UAMIZ); G. B. Hinton 17227 (UAMIZ); H. H. Iltis 75 (UAMIZ); A. R. López-Ferrari 2214 (UAMIZ); D. Martínez A. 6911-B (UAMIZ); L. Sánchez S. 483 (UAMIZ); J. Vázquez 14 (MEXU).

Stenorrhynchos lanceolatum (Aubl.) Rich. ex Spreng: A. Espejo 3306 (UAMIZ), 3624-b (UAMIZ); J. García-Cruz 1006 (UAMIZ); A. R. López-Ferrari 2460 (UAMIZ); C. G. Pringle 13215 (MEXU); J. Vázquez 1680 (MEXU).

*Stenorrhynchos michuacanum (Lex.) Lindl.: A. Espejo 5943 (UAMIZ); A. R. López-Ferrari 2461 (UAMIZ).

*Stenorrhynchos sulphureum (Lex.) Lindl.: E. Lyonnet 1526 (MEXU).

Triphora trianthophora (Sw.) Rydb.: A. Espejo 5585 (UAMIZ); J. García-Cruz 916 (UAMIZ).

\section{POACEAE}

Aegopogon cenchroides Humb. \& Bonpl. ex Willd.: G. Ayala A. 81 (MEXU); V. L. Cardoso 1369 (HUMO, MEXU); E. Lyonnet 626 (MEXU); M. A. Panti M. 380 (HUMO).

Aegopogon tenellus (DC.) Trin.: R. Cerros 1605 (UAMIZ); J. Espinosa 259 (MEXU); M. Ishiki 757 (MEXU); F. Sánchez E. s.n. (MEXU); M. Sánchez s.n. (MEXU); J. Vázquez Aguilar s.n. (MEXU).

Agrostis bourgaei E. Fourn.: E. Lyonnet 2518 (MEXU); P. R. Matosic y V. L. Cardoso 1274 (MEXU).

Agrostis ghiesbreghtii E. Fourn.: E. Matuda 26005 (MEXU).

Agrostis perennans (Walter) Tuck.: V. L. Cardoso s.n. (MEXU); E. Lyonnet 287089 (MEXU); E. Matuda s.n. (MEXU); J. Vázquez s.n. (UAMIZ).

Agrostis schaffneri E. Fourn.: N. C. Fassett 28438 (MEXU); E. Lyonnet 291285 (MEXU). Agrostis tolucensis Kunth: E. Lyonnet 2497 (MEXU).

Aristida appressa Vasey: E. Lyonnet 2428 (MEXU). Aristida schiedeana Trin. \& Rupr.: R. Cerros 103 (UAMIZ); E. Lyonnet 633 (MEXU). Blepharoneuron tricholepis (Torr.) Nash: E. Lyonnet 45 (MEXU).

Botriochloa barbinodis (Lag.) Herter var. perforata (Trin. ex E. Fourn.) Gould: R. Cerros 1616 (UAMIZ). 
Bothriochloa wrightii (Hack.) Henrard: E. Lyonnet 1861 (MEXU).

Bouteloua curtipendula (Michx.) Torr. var. tenuis Gould \& Kapadia: R. Cerros 162 (UAMIZ); P. Dávila s.n. (MEXU).

Bouteloua radicosa (E. Fourn.) Griffiths: E. L. Estrada 1813 (MEXU); E. Matuda 26320 (MEXU).

Bouteloua repens (Kunth) Scribn. \& Merr.: R. Cerros 131 (UAMIZ); M. Núñez M. 9 (MEXU); J. M. Olascoaga 114 (MEXU).

Brachypodium mexicanum (Roem. \& Schult.) Link: E. Lyonnet 2509 (ENCB); J. R. Reeder 3043 (MEXU).

Bromus anomalus Rupr. ex E. Fourn.: E. Lyonnet 2495 (MEXU).

Bromus carinatus Hook. \& Arn.: J. J. Ortiz 1134 (UAMIZ); A. Pulido 18 (UAMIZ), 80 (UAMIZ); A. Sotelo G. 352 (MEXU).

Bromus exaltatus Bernh.: E. Lyonnet 1811 (ENCB, MEXU); E. Matuda 25587 (MEXU).

Calamagrostis orizabae Steud.: V. L. Cardoso 1542 (UAMIZ, HUMO); J. Rzedowski 36181 (UAMIZ).

Cenchrus echinatus L.: E. L. Estrada 1190 (MEXU).

Chaetium bromoides (J. Presl) Benth. ex Hemsl.: A. Díaz 19 (MEXU); E. Lyonnet 2447 (MEXU); E. Matuda 25920 (MEXU).

Chloris virgata Sw.: M. C. Campos 73 (HUMO).

Cinna poaeformis (Kunth) Scribn. \& Merr.: H. H. Iltis 121 (MEXU); E. Lyonnet 229 (MEXU), 2520 (MEXU); A. Pulido 52 (UAMIZ), 65 (UAMIZ).

Deschampsia elongata (Hook.) Munro: E. Lyonnet 2459 (MEXU).

Echinochloa crusgalli (L.) P. Beauv. var. mitis (Pursh) Peterm.: E. Matuda s.n. (MEXU).

Eragrostis mexicana (Hornem.) Link: N. Espino V. 17 (MEXU); A. Pulido 19 (UAMIZ); J.

T. Villamil s.n. (MEXU).

Festuca amplissima E. Fourn.: G. Andrade s.n. (MEXU); I. Escamilla G. 23 (MEXU); E. Lyonnet 2482 (MEXU); C. R. Orcutt 3743 (MEXU).

Festuca hephaestophila Nees ex Steud.: V. P. Mitchell 56 (MEXU).

Festuca orizabensis E.B. Alexeev: V. L. Cardoso 21 (MEXU).

Festuca rosei Piper: I. Díaz V. 1147 (MEXU); E. Lyonnet 2471 (MEXU); E. Matuda 25612 (MEXU).

Festuca tolucensis Kunth: H. H. Iltis 189 (MEXU).

Glyceria striata (Lam.) Hitchc.: Adams 44 (MEXU); J. Bonilla 1485 (HUMO, MEXU), 1508 (HUMO, MEXU), 1517 (HUMO, MEXU), 1524 (HUMO, MEXU), 1537 (HUMO, MEXU), 1561 (HUMO, MEXU); V. L. Cardoso 1246 (MEXU).

Heteropogon contortus (L.) P. Beauv. ex Roem. \& Schult.: R. Cerros 160 (UAMIZ).

Hierochloë mexicana (Rupr. ex E. Fourn.) Benth. ex Hitchc.: E. Matuda 25594 (MEXU).

Lasiacis divaricata (L.) Hitchc.: M. Sánchez s.n. (MEXU).

Lasiacis nigra Davidse: E. Lyonnet 2541 (MEXU); F. Miranda 1591 (MEXU).

Leersia hexandra Sw.: N. Espino V. 20 (MEXU).

Microchloa kunthii Desv.: R. Cerros 123 (UAMIZ).

Muhlenbergia diversiglumis Trin.: C. G. Pringle 1175 MEXU), s .n. (MEXU).

Muhlenbergia emersleyi Vasey: E. Lyonnet 1830 (ENCB), 2873 (MEXU); J. F. Zúñiga s.n. (MEXU). 
Muhlenbergia glabrata (Kunth) Trin.: E. Manrique 1242 (MEXU).

Muhlenbergia implicata (Kunth) Trin.: R. Cerros 1601 (UAMIZ).

Muhlenbergia macroura (Kunth) Hitchc.: I. Escamilla 24 (MEXU); E. Lyonnet 56 (MEXU); E. Matuda 51 (MEXU); J. J. Ortiz 1128 (MEXU); A. Pulido 2 (UAMIZ), 34 (UAMIZ); F. Sánchez E. s. n. (MEXU).

Muhlenbergia microsperma (DC.) Trin.: G. Ayala A. 76 (MEXU).

Muhlenbergia nigra Hitchc.: I. Escamilla 28 (MEXU); E. Lyonnet 1408 (MEXU).

Muhlenbergia peruviana (P. Beauv.) Steud.: E. Matuda 21949 (MEXU).

Muhlenbergia quadridentata (Kunth) Trin.: A. Pulido 2496 (MEXU)

Muhlenbergia robusta (E. Fourn.) Hitchc.: A. Pulido 8 (UAMIZ); E. Lyonnet 2553 (MEXU).

Muhlenbergia tenella (Kunth) Trin.: R. Cerros 1608 (UAMIZ).

Nassella mucronata (Kunth) R.W. Pohl: I. Díaz V. 1121 (MEXU).

Oplismenus burmannii (Retz.) P. Beauv.: T. Estrada 296 (ENCB, MEXU); J. Vázquez 3440 (MEXU).

Oplismenus compositus (L.) P. Beauv. var. rariflorus (J. Presl) U. Scholz: E. Lyonnet 259 (MEXU).

Panicum commutatum Schult.: E. Lyonnet 242262 (MEXU), 242272 (MEXU).

Paspalum candidum (Humb. \& Bonpl. ex Flüggé) Kunth: A. Pulido 81 (UAMIZ).

Paspalum convexum Humb. \& Bonpl. ex Flüggé: R. Cerros 1598 (UAMIZ); N. Espino V. 13

(MEXU); A. Pulido 81 (UAMIZ); J. Vázquez 2634 (MEXU).

Paspalum humboldtianum Flüggé: E. Lyonnet 510900036 (MEXU); F. Miranda 3790

(MEXU); J. N. Rose 10203 (MEXU).

Paspalum notatum Flüggé: E. Lyonnet 2432 (MEXU); D. Martínez A. 315 (MEXU).

Paspalum plicatum Michx.: N. Espino V. 19 (MEXU).

Paspalum squamulatum E. Fourn.: E. Lyonnet 2434 (MEXU); E. Matuda 25919 (MEXU).

Paspalum tenellum Willd.: J. R. Reeder 2212 (MEXU).

Piptochaetium fimbriatum (Kunth) Hitchc.: C. J. Carney 59 (MEXU); E. Lyonnet 2512 (MEXU); J. J. Ortiz 1132 (ENCB, MEXU).

Piptochaetium seleri (Pilg.) Henrard: E. Lyonnet 2511 (MEXU).

Piptochaetium virescens (Kunth) Parodi: R. F. Adams 50 (MEXU); L. B. Cole 56 (MEXU); I. Díaz V. 1061 (MEXU); H. H. Iltis 921 (MEXU); E. Lyonnet 2498 (MEXU), 287144 (MEXU); V. P. Mitchell 65 (MEXU); W. T. Smith 60 (MEXU).

Schizachyrium sanguineum (Retz.) Alston var. brevipedicellatum (Beal) S.L. Hatch: J. Espinosa 213 (MEXU); J. Rzedowski 19149 (ENCB, MEXU).

Setaria parviflora (Poir.) Kerguélen: C. R. Orcutt 3861 (MEXU); D. Pacheco 61 (MEXU); A. Pulido 1 (UAMIZ), 21 (UAMIZ).

Sorghastrum incompletum (J. Presl) Nash: E. Lyonnet 1838 (MEXU).

Sporobolus indicus (L.) R. Br.: A. Chimal s.n. (MEXU); M. Gutiérrez 405 (MEXU); E. Lyonnet 1810 (MEXU); D. Martinez A. s.n. (MEXU).

Stipa ichu (Ruiz \& Pav.) Kunth: G. Ayala A. 46 (MEXU); I. Escamilla 19 (MEXU); G. Flores C. 7597 (MEXU); P. R. Matosic 1273 (HUMO, MEXU); G. M. Reyes 1038 (MEXU); F. Sánchez E. s.n. (MEXU).

Trachypogon plumosus (Humb. \& Bonpl. ex Willd.) Nees: E. Lyonnet 628 (MEXU). 
Triniochloa stipoides (Kunth) Hitchc.: E. Lyonnet 630 (MEXU); 1535 (MEXU).

Trisetum deyeuxioides (Kunth) Kunth: J. J. Ortiz 1131 (MEXU).

Trisetum irazuense (Kuntze) Hitchc.: E. Lyonnet 2503 (MEXU).

Trisetum virletii E. Fourn.: R. Bye 19092 (MEXU); G. B. Hinton 17531-bis (MEXU); E. Lyonnet 3210 (MEXU); E. Matuda s.n. (MEXU); A. Pulido 6 (UAMIZ), 15 (UAMIZ); L. Silva 95 (MEXU); J. Vázquez 2354 (MEXU).

Urochloa plantaginea (Link) R.D. Webster: N. Espino V. 11 (MEXU), 16 (MEXU); G. Gándara 28 (MEXU); E. Lyonnet 2530 (MEXU).

PONTEDERIACEAE

Heteranthera peduncularis Benth.: J. Bonilla 337 (MEXU); J. Ceja 83 (UAMIZ).

POTAMOGETONACEAE

Potamogeton crispus L.: J. Bonilla 377 (HUMO).

Potamogeton illinoensis Morong: Brigada de Vegetación Acuática 164 (MEXU); E. Gallegos y M. González 1 (MEXU).

Potamogeton pusillus L.: J. Bonilla 345 (HUMO, MEXU), 378 (HUMO, MEXU), 478 (HUMO, MEXU); N. C. Fassett 28 (MEXU); E. Gallegos 2 (MEXU); M. González G. 16 (MEXU); A. Lot-Helgueras 1201 (MEXU); M. Ulloa S. s.n. (MEXU).

SMILACACEAE

*Smilax cordifolia Humb. \& Bonpl. ex Willd.: F. Miranda 170 (MEXU).

Smilax jalapensis Schltdl.: E. Lyonnet 51100004 (MEXU).

*Smilax moranensis M. Martens \& Galeotti: J. Bonilla 580 (HUMO), 638 (HUMO), 647 (HUMO); I. Díaz V. 1091 (MEXU); R. Hernández M. 4256 (MEXU); M. Peña s.n. (MEXU); C. G. Pringle s.n. (MEXU); A. Pulido 58 (UAMIZ), 75 (UAMIZ).

*Smilax pringlei Greenm.: J. Ceja 801 (UAMIZ); A. Espejo 2640 (UAMIZ), 3557 (UAMIZ), 5421 (UAMIZ), 5579 (UAMIZ); G. B. Hinton 17099 (ENCB, MEXU); S. D. Koch 7932 (MEXU); A. R. López-Ferrari 2168 (UAMIZ), 2467 (UAMIZ), 2725 (UAMIZ); I. Luna 228 (MEXU).

TYPHACEAE

Typha latifolia L.: R. Hernández 187 (ENCB). 